\title{
Application of a volume-translated Peng-Robinson equation of state on vapor-liquid equilibrium calculations
}

\author{
Jung-Chin Tsai, Yan-Ping Chen * \\ Department of Chemical Engineering, National Taiwan Unicersity. Taipei. Tainan \\ Received 23 June 1997; accepted 30 November 1997
}

\begin{abstract}
A volume-translated Peng-Robinson (VTPR) equation of state (EOS) is developed in this study. Besides the two parameters in the original Peng-Robinson equation of state, a volume correction term is employed in the VTPR EOS. In this equation, the temperature dependence of the EOS energy parameter was regressed by an improved expression which yields better correlation of pure-fluid vapor pressures. The volume correction parameter is also correlated as a function of the reduced temperature. The VTPR EOS includes two optimally fitted parameters for each pure fluid. These parameters are reported for over 100 nonpolar and polar components. The VTPR EOS shows satisfactory results in calculating the vapor pressures and both the saturated vapor and liquid molar volumes. In comparison with other commonly used cubic EOS, the VTPR EOS presents better results. especially for the saturated liquid molar volumes of polar systems. VLE calculations on fluid mixtures were also studied in this work. Traditional van der Waals one-fluid mixing rules and other mixing models using excess free energy equations were employed in the new EOS. The VTPR EOS is comparable to other EOS in VLE calculations with various mixing rules, but yields better predictions on the molar volumes of liquid mixtures. (C) 1998 Elsevier Science B.V. All rights reserved.
\end{abstract}

Keywords: Equation of state: Volume translation; Nonpolar component: Polar component; Vapor-liquid equilibria

\section{Introduction}

Modifications of equations of state are important to the design and simulation of chemical processes. Taking the cubic-type EOS for example, many efforts have been devoted to improve the temperature dependence of the energy parameter (e.g., Refs. [1-3]). This approach results in accurate

\footnotetext{
* Corresponding author.
} 
Table 1

Calculated results of saturated vapor pressures and liquid molar volumes of nonpolar and polar pure fluids using the VTPR equation of state

\begin{tabular}{|c|c|c|c|c|c|c|c|c|c|}
\hline \multirow[t]{2}{*}{ Compounds } & \multicolumn{2}{|c|}{ Temperature range $(\mathrm{K})$} & \multicolumn{2}{|c|}{ Data points } & \multirow[t]{2}{*}{$N$} & \multirow[t]{2}{*}{$k_{3}$} & \multicolumn{2}{|c|}{ This work } & \multirow[t]{2}{*}{ Data sources } \\
\hline & $\overline{P^{\mathrm{V}}}$ & $V^{\mathrm{L}}$ & $\overline{P^{V}}$ & $\overline{V^{\mathrm{L}}}$ & & & $\begin{array}{l}\text { AAD } \\
(P) \%\end{array}$ & $\begin{array}{l}\text { AAD } \\
\left(V^{L}\right) \%\end{array}$ & \\
\hline Argon & $84-149$ & $84-148$ & 68 & 67 & 0.03877 & 0.25486 & 0.70 & 0.90 & [14] \\
\hline Krypton & $116-208$ & $116-206$ & 47 & 46 & 0.05519 & 0.21040 & 0.64 & 1.00 & {$[14]$} \\
\hline Oxygen & $66-152$ & $72-152$ & 88 & 82 & 0.09137 & 0.25742 & 0.85 & 1.02 & [14] \\
\hline Nitrogen & $63-125$ & $63-124$ & 64 & 63 & 0.09967 & 0.24086 & 0.46 & 1.01 & [14] \\
\hline $\begin{array}{l}\text { Carbon } \\
\text { dioxide }\end{array}$ & $217-302$ & $217-298$ & 42 & 33 & 0.11333 & 0.28996 & 0.29 & 1.46 & {$[14]$} \\
\hline $\begin{array}{l}\text { Sulfur } \\
\text { dioxide }\end{array}$ & $203-428$ & $338-423$ & 31 & 15 & 0.21833 & 0.26345 & 1.14 & 1.02 & {$[14]$} \\
\hline $\begin{array}{l}\text { Hydrogen } \\
\text { chloride }\end{array}$ & $159-240$ & $170-230$ & 11 & 8 & 0.07253 & 0.07685 & 0.89 & 0.05 & {$[15]$} \\
\hline Ammonia & $200-406$ & $200-360$ & 22 & 16 & 0.15327 & 0.01978 & 0.40 & 1.07 & [14] \\
\hline Methane & $91-190$ & $91-187$ & 45 & 44 & 0.08248 & 0.20978 & 0.53 & 1.19 & {$[16]$} \\
\hline Ethane & $163-304$ & $163-295$ & 30 & 28 & 0.11292 & 0.20077 & 0.47 & 0.89 & {$[16]$} \\
\hline Propane & $173-357$ & $173-357$ & 30 & 30 & 0.16846 & 0.22685 & 0.84 & 1.05 & [16] \\
\hline Butane & $217-392$ & $217-392$ & 28 & 28 & 0.15546 & 0.25370 & 0.83 & 0.55 & {$[16]$} \\
\hline Isobutane & $200-401$ & $213-369$ & 32 & 25 & 0.18381 & 0.25559 & 1.07 & 0.47 & [16] \\
\hline Pentane & $240-454$ & $240-454$ & 30 & 30 & 0.16851 & 0.26392 & 0.77 & 0.94 & [16] \\
\hline $\begin{array}{l}\text { 2-Methyl } \\
\text { butane }\end{array}$ & $236-447$ & $236-440$ & 29 & 28 & 0.14908 & 0.27640 & 0.58 & 0.96 & {$[16]$} \\
\hline $\begin{array}{l}\text { 2,2-Dimethyl } \\
\text { propane }\end{array}$ & $257-433$ & $261-425$ & 45 & 42 & 0.14564 & -0.27786 & 0.46 & 1.37 & {$[16]$} \\
\hline Hexane & $282-492$ & $267-492$ & 28 & 31 & 0.16397 & 0.27558 & 1.05 & 1.06 & {$[16]$} \\
\hline $\begin{array}{l}\text { 2-Methyl } \\
\text { pentane }\end{array}$ & $289-497$ & $289-480$ & 25 & 22 & 0.15545 & 0.27999 & 0.50 & 1.01 & {$[16]$} \\
\hline $\begin{array}{l}\text { 3-Methyl } \\
\text { pentane }\end{array}$ & $283-504$ & $273-494$ & 43 & 42 & 0.16917 & 0.28046 & 0.78 & 1.09 & {$[16]$} \\
\hline $\begin{array}{l}\text { 2,2-Dimethyl } \\
\text { butane }\end{array}$ & $236-488$ & $274-463$ & 41 & 31 & 0.17424 & 0.27632 & 0.84 & 0.68 & {$[16]$} \\
\hline $\begin{array}{l}\text { 2,3-Dimethyl } \\
\text { butane }\end{array}$ & $259-499$ & $275-489$ & 45 & 39 & 0.16302 & 0.27750 & 0.74 & 1.04 & {$[16]$} \\
\hline Heptane & $304-507$ & $280-523$ & 25 & 31 & 0.17822 & 0.26154 & 1.17 & 1.08 & {$[16]$} \\
\hline $\begin{array}{l}\text { 2-Methyl } \\
\text { hexane }\end{array}$ & $284-524$ & $284-512$ & 41 & 40 & 0.19917 & 0.28434 & 0.67 & 1.16 & {$[16]$} \\
\hline $\begin{array}{l}\text { 3-Methyl } \\
\text { hexane }\end{array}$ & $283-529$ & $288-518$ & 44 & 41 & 0.19433 & 0.27525 & 0.52 & 1.15 & {$[16]$} \\
\hline $\begin{array}{l}\text { 3-Ethyl } \\
\text { pentane }\end{array}$ & $300-532$ & $300-523$ & 28 & 26 & 0.18450 & 0.29840 & 0.64 & 1.15 & {$[16]$} \\
\hline $\begin{array}{l}\text { 2,2-Dimethyl } \\
\text { pentane }\end{array}$ & $286-512$ & $286-503$ & 28 & 27 & 0.18038 & 0.29821 & 0.67 & 0.70 & {$[16]$} \\
\hline $\begin{array}{l}\text { 2,3-Dimethyl } \\
\text { pentane }\end{array}$ & $260-529$ & $297-522$ & 37 & 30 & 0.20322 & 0.30495 & 0.58 & 1.02 & [16] \\
\hline $\begin{array}{l}2,2,3 \text {-Trimethy } \\
\text { butane }\end{array}$ & $290-525$ & $273-513$ & 41 & 42 & 0.16463 & 0.30112 & 0.64 & 0.93 & {$[16]$} \\
\hline
\end{tabular}


Table 1 (continued)

\begin{tabular}{|c|c|c|c|c|c|c|c|c|c|}
\hline \multirow[t]{2}{*}{ Compounds } & \multicolumn{2}{|c|}{ Temperature range $(\mathrm{K})$} & \multicolumn{2}{|c|}{ Data points } & \multirow[t]{2}{*}{$N$} & \multirow[t]{2}{*}{$k_{3}$} & \multicolumn{2}{|c|}{ This work } & \multirow[t]{2}{*}{ Data sources } \\
\hline & $P^{\mathrm{V}}$ & $V^{\mathrm{L}}$ & $\overline{P^{v}}$ & $V^{1}$ & & & $\begin{array}{l}\mathrm{AAD} \\
(P) \%\end{array}$ & $\begin{array}{l}\mathrm{AAD} \\
\left(V^{\mathrm{L}}\right) \%\end{array}$ & \\
\hline $\begin{array}{l}\text { 2,2,4-Trimethyl } \\
\text { pentane }\end{array}$ & $291-535$ & $291-527$ & 30 & 29 & 0.18330 & 0.31944 & 0.90 & 1.01 & {$[16]$} \\
\hline Octane & $320-544$ & $312-552$ & 29 & 30 & 0.19773 & 0.24145 & 0.98 & 1.06 & [16] \\
\hline$n$-Decane & $283-588$ & $333-443$ & 44 & 12 & 0.25192 & 0.17726 & 1.20 & 0.28 & {$[16-19]$} \\
\hline$n$-Undecane & $348-499$ & $353-473$ & 30 & 13 & 0.24055 & 0.13471 & 0.61 & 0.53 & {$[16,18,20]$} \\
\hline$n$-Dodecane & $353-588$ & $373-483$ & 13 & 12 & 0.28162 & 0.08256 & 0.91 & 0.51 & {$[16-18]$} \\
\hline n-Tridecane & $380-540$ & $393-513$ & 30 & 13 & 0.23996 & 0.03898 & 0.81 & 0.62 & {$[16,18,20]$} \\
\hline$n$-Tetradecane & $373-588$ & $413-523$ & 16 & 12 & 0.29092 & -0.02270 & 0.74 & 0.40 & {$[16-18]$} \\
\hline n-Pentadecane & $435-577$ & $423-543$ & 28 & 13 & 0.17570 & -0.06416 & 0.80 & 0.42 & {$[16,18,20]$} \\
\hline n-Hexadecane & $393-583$ & $443-553$ & 20 & 12 & 0.32062 & -0.12198 & 0.60 & 0.29 & {$[16,17,18,20]$} \\
\hline$n$-Heptadecane & $461-609$ & $453-573$ & 28 & 13 & 0.21106 & -0.18978 & 0.84 & 0.57 & {$[18,20]$} \\
\hline n-Octadecane & $413-588$ & $473-583$ & 17 & 12 & 0.33351 & -0.19293 & 0.74 & 0.60 & {$[17,18]$} \\
\hline$n$-Nonadecane & $423-588$ & $463-593$ & 16 & 15 & 0.34965 & -0.20808 & 1.10 & 0.74 & {$[17,18]$} \\
\hline$n$-Eicosane & $388-583$ & $473-613$ & 41 & 15 & 0.39354 & -0.20140 & 1.76 & 0.78 & {$[17-19]$} \\
\hline n-Docosane & $453-523$ & $473-750$ & 12 & 33 & 0.44472 & 0.02619 & 1.89 & 1.21 & {$[17,18]$} \\
\hline$n$-Tetracosane & $453-533$ & $460-774$ & 9 & 36 & 0.51055 & 0.26662 & 1.68 & 1.69 & {$[17,18]$} \\
\hline n-Octacosane & $503-533$ & $535-635$ & 10 & 13 & 0.76902 & 1.85456 & 1.87 & 1.23 & {$[17-19]$} \\
\hline Ethene & $120-281$ & $128-277$ & 41 & 37 & 0.14312 & 0.19916 & 0.86 & 1.06 & {$[16]$} \\
\hline Propene & $163-364$ & $176-364$ & 33 & 30 & 0.15774 & 0.25091 & 0.87 & 1.19 & [16] \\
\hline 1-Butene & $215-413$ & $215-388$ & 40 & 35 & 0.14186 & 0.24347 & 0.56 & 0.77 & [16] \\
\hline 1-Pentene & $221-46.3$ & $221-443$ & 37 & 34 & 0.18205 & 0.23578 & 0.84 & 0.94 & {$[16]$} \\
\hline 1-Hexene & $283-353$ & $283-334$ & 33 & 45 & 0.18829 & 0.23050 & 0.14 & 0.10 & {$[16]$} \\
\hline Benzene & $284-543$ & $284-543$ & 41 & 41 & 0.19292 & 0.22169 & 0.90 & 0.90 & {$[16]$} \\
\hline Ethylbenzene & $297-616$ & $297-487$ & 33 & 20 & 0.19837 & 0.24148 & 0.88 & 0.57 & {$[16]$} \\
\hline Toluene & $301-546$ & $282-546$ & 27 & 29 & 0.16898 & 0.28740 & 0.93 & 0.92 & {$[16]$} \\
\hline o-Xylene & $303-630$ & $312-612$ & 38 & 35 & 0.18030 & 0.25739 & 0.98 & 1.11 & [16] \\
\hline$m$-Xylene & $302-616$ & $302-582$ & 37 & 33 & 0.17577 & 0.22372 & 0.79 & 0.78 & {$[16]$} \\
\hline$p$-Xylenc & $296-616$ & $296-491$ & 42 & 26 & 0.17247 & 0.21869 & 0.97 & 0.52 & {$[16]$} \\
\hline Cyclopropane & $203-397$ & $197-386$ & 35 & 32 & 0.12271 & 0.18443 & 0.96 & 1.41 & {$[16]$} \\
\hline Cyclobutane & $203-285$ & $203-273$ & 41 & 35 & 0.13612 & 0.29062 & 0.54 & 0.97 & {$[16]$} \\
\hline Cyclopentane & $229-510$ & $229-496$ & 40 & 38 & 0.18433 & 0.26814 & 0.81 & 1.28 & {$[16]$} \\
\hline Cyclohexane & $279-541$ & $279-535$ & 43 & 42 & 0.19138 & 0.26575 & 0.83 & 0.94 & {$[16]$} \\
\hline Cyclobeptane & $28.3-604$ & $297-575$ & 45 & 39 & 0.19322 & 0.30608 & 0.55 & 0.96 & {$[16]$} \\
\hline Propyne & $217-401$ & $217-387$ & 40 & 37 & 0.16686 & 0.23182 & 0.80 & 0.79 & [16] \\
\hline l-Butync & $212-282$ & $242-282$ & 36 & 21 & 0.50745 & 0.03694 & 1.19 & 0.05 & {$[16]$} \\
\hline Fluoromethane & $132-288$ & $144-292$ & 38 & 34 & 0.12077 & -0.03299 & 0.57 & 1.16 & {$[16]$} \\
\hline $\begin{array}{l}\text { Dichlorofluoro } \\
\text { methane }\end{array}$ & $245-450$ & $207-439$ & 38 & 42 & 0.20585 & 0.23148 & 0.61 & 1.0 .3 & {$[16]$} \\
\hline $\begin{array}{l}\text { Trifluoro } \\
\text { methane }\end{array}$ & $141-298$ & $191-298$ & 45 & 27 & 0.15225 & 0.16622 & 0.86 & 0.99 & {$[16]$} \\
\hline
\end{tabular}

$\operatorname{AAD}(\%)=\frac{1(\%)}{n} \sum\left[\left|X^{\exp }-X^{\mathrm{cal}}\right| / X^{\mathrm{exp}}\right] ; X=P$ or $V^{\mathrm{L}}$.

prediction of saturated vapor pressures. It, however, does not necessarily yield the accurate volumetric behavior of fluids and their mixtures. On the other hand, a volume translation can be adopted in the EOS. Martin [4] has pointed out that with the volume translation, an EOS can be made more realistic. 
Table 2

Calculated results of saturated vapor pressures and liquid molar volumes of nonpolar and polar pure fluids using the VTPR equation of state

\begin{tabular}{|c|c|c|c|c|c|c|c|c|c|}
\hline \multirow[t]{2}{*}{$\overline{\text { Compounds }}$} & \multicolumn{2}{|c|}{ Temperature range $(\mathrm{K})$} & \multicolumn{2}{|c|}{ Data points $N$} & & \multirow[t]{2}{*}{$k_{3}$} & \multicolumn{2}{|c|}{ This work } & \multirow{2}{*}{$\begin{array}{l}\text { Data } \\
\text { sources }\end{array}$} \\
\hline & $\overline{P^{V}}$ & $V^{\mathrm{L}}$ & $\overline{P^{V}}$ & $V^{\mathrm{L}}$ & & & $\begin{array}{l}\text { AAD } \\
(P) \%\end{array}$ & $\begin{array}{l}\text { AAD } \\
\left(V^{L}\right) \%\end{array}$ & \\
\hline Trichloromethane & $244-431$ & $259-524$ & 27 & 38 & 0.16945 & 0.31307 & 0.76 & 0.98 & {$[16]$} \\
\hline Dichloromethane & $220-503$ & $212-414$ & 39 & 28 & 0.25264 & 0.10812 & 0.92 & 0.49 & [16] \\
\hline Tetrafluoromethane & $89-227$ & $107-183$ & 45 & 25 & 0.17605 & 0.31695 & 0.97 & 0.91 & [16] \\
\hline Trichlorofluoromethane & $226-465$ & $219-465$ & 37 & 38 & 0.16940 & 0.26791 & 0.72 & 1.30 & {$[16]$} \\
\hline Dichlorodifluoromethane & $177-383$ & $182-378$ & 40 & 38 & 0.16366 & 0.26461 & 0.92 & 1.21 & [16] \\
\hline Chloromethane & $193-398$ & $193-398$ & 40 & 39 & 0.14825 & 0.11442 & 0.55 & 1.00 & {$[16]$} \\
\hline Chlorodifluoromethane & $183-365$ & $203-357$ & 45 & 38 & 0.17359 & 0.20674 & 0.50 & 1.10 & {$[16]$} \\
\hline 1,1-Difluoroethane & $215-382$ & $232-357$ & 44 & 31 & 0.01995 & 0.09867 & 1.42 & 0.93 & {$[16]$} \\
\hline $\begin{array}{l}\text { 1,2-Dichloro-1,1,2,2-tetra } \\
\text { fluoroethane }\end{array}$ & $245-413$ & $214-403$ & 31 & 38 & 0.21240 & 0.32566 & 1.15 & 1.07 & {$[16]$} \\
\hline Chloroethane & $217-460$ & $244-383$ & 45 & 19 & 0.13881 & 0.07090 & 0.78 & 1.23 & {$[16]$} \\
\hline 1,2-Dichloroethane & $279-403$ & $261-349$ & 35 & 25 & 0.12569 & 0.26278 & 0.83 & 1.03 & {$[16]$} \\
\hline Fluoroethane & $169-375$ & $173-234$ & 45 & 14 & 0.13388 & 0.13826 & 0.32 & 0.77 & {$[16]$} \\
\hline $\begin{array}{l}\text { 1,1,2-Trichloro-1,2,2-tri } \\
\text { fluoroethane }\end{array}$ & $304-482$ & $281-465$ & 33 & 34 & 0.16091 & 0.31131 & 0.23 & 1.04 & {$[16]$} \\
\hline 1,1,1-Trifluoroethane & $173-346$ & $200-315$ & 45 & 26 & 0.17242 & 0.12161 & 0.32 & 1.45 & {$[16]$} \\
\hline 1,1,1-Trichloroethane & $251-371$ & $243-336$ & 42 & 33 & 0.19610 & 0.23948 & 0.63 & 1.10 & {$[16]$} \\
\hline Methanol & $235-511$ & $220-474$ & 34 & 28 & 0.03221 & -0.04426 & 1.45 & 1.38 & [16] \\
\hline Ethanol & $271-511$ & $239-463$ & 31 & 26 & 0.20761 & 0.02687 & 0.73 & 1.17 & {$[16]$} \\
\hline 1-Propanol & $280-525$ & $280-492$ & 30 & 26 & 0.40666 & 0.11811 & 0.72 & $1.0 \mathrm{I}$ & {$[16]$} \\
\hline 2-propanol & $279-480$ & $265-376$ & 30 & 17 & 0.45120 & 0.04776 & 0.64 & 0.08 & {$[16]$} \\
\hline 1-Butanol & $295-556$ & $295-523$ & 32 & 31 & 0.54197 & 0.16820 & 0.41 & 0.75 & [16] \\
\hline 2-Butanol & $281-525$ & $281-488$ & 34 & 29 & 0.61833 & 0.15436 & 0.60 & 0.54 & {$[16]$} \\
\hline 2-Methyl-1-propanol & $300-543$ & $300-526$ & 29 & 26 & 0.55775 & 0.16725 & 0.52 & 1.19 & {$[16]$} \\
\hline 2-Methyl-2-propanol & $304-443$ & $304-443$ & 35 & 35 & 0.64017 & 0.14725 & 0.37 & 0.28 & {$[16]$} \\
\hline 1-Pentanol & $319-401$ & $336-557$ & 11 & 28 & 0.66083 & 0.19857 & 0.27 & 0.71 & {$[16]$} \\
\hline 1-Hexanol & $324-428$ & $310-532$ & 9 & 31 & 0.72399 & 0.22577 & 1.12 & 0.92 & [16] \\
\hline 1-Heptanol & $351-445$ & $351-595$ & 12 & 32 & 0.68053 & 0.22027 & 1.10 & 0.72 & {$[16]$} \\
\hline 1-Octanol & $372-524$ & $364-617$ & 18 & 31 & 0.57262 & 0.19203 & 1.44 & 1.09 & [16] \\
\hline 1-Nonanol & $366-481$ & $366-653$ & 12 & 36 & 1.00074 & 0.19903 & 1.03 & 0.94 & [16] \\
\hline I-Decanol & $379-509$ & $327-622$ & 13 & 33 & 0.53213 & 0.07796 & 1.54 & 1.09 & {$[16]$} \\
\hline Phenol & $391-446$ & $368-449$ & 18 & 26 & 0.15996 & 0.50681 & 1.05 & 0.89 & {$[16]$} \\
\hline Cyclohexanol & $364-489$ & $305-430$ & 18 & 18 & 0.45851 & 0.06946 & 1.18 & 0.19 & {$[16]$} \\
\hline Acetone & $253-508$ & $253-463$ & 35 & 26 & 0.14457 & 0.05648 & 0.66 & 1.20 & [16] \\
\hline 2-Butanone & $272-536$ & $272-319$ & 35 & 7 & 0.16786 & 0.14610 & 0.85 & 0.17 & {$[16]$} \\
\hline 2-Pentanone & $282-353$ & $282-353$ & 11 & 11 & 0.19851 & 0.18962 & 0.85 & 0.25 & {$[16]$} \\
\hline 3-Pentanone & $285-544$ & $285-347$ & 43 & 11 & 0.22913 & 0.18669 & 0.27 & 0.30 & {$[16]$} \\
\hline 3-Methyl-2-butanone & $276-500$ & $288-319$ & 37 & 6 & 0.18722 & 0.25482 & 0.62 & 0.53 & {$[16]$} \\
\hline 2-Hexanone & $299-427$ & $299-332$ & 40 & 11 & 0.19036 & 0.23240 & 0.70 & 0.35 & {$[16]$} \\
\hline 3-Hexanone & $312-394$ & $299-324$ & 34 & 11 & 0.20545 & 0.22667 & 0.53 & 0.25 & {$[16]$} \\
\hline 3,3-Dimethyl-2-butanone & $295-405$ & $295-353$ & 41 & 22 & 0.19793 & 0.31412 & 0.58 & 0.53 & {$[16]$} \\
\hline 2-Heptanone & $335-432$ & $310-408$ & 25 & 25 & 0.03938 & 0.47751 & 0.90 & 0.86 & {$[16]$} \\
\hline 5-Nonanone & $324-485$ & $301-356$ & 36 & 13 & 0.24216 & 0.08138 & 0.82 & 0.18 & {$[16]$} \\
\hline 4-Methyl-2-pentanone & $286-500$ & $286-373$ & 33 & 14 & 0.25880 & 0.23928 & 0.96 & 0.52 & {$[16]$} \\
\hline
\end{tabular}


Table 2 (continued)

\begin{tabular}{|c|c|c|c|c|c|c|c|c|c|}
\hline \multirow[t]{2}{*}{$\overline{\text { Compounds }}$} & \multicolumn{2}{|c|}{ Temperature range $(\mathrm{K})$} & \multicolumn{2}{|c|}{ Data points } & \multirow[t]{2}{*}{$N$} & \multirow[t]{2}{*}{$k_{3}$} & \multicolumn{2}{|c|}{ This work } & \multirow{2}{*}{$\begin{array}{l}\text { Data } \\
\text { sources }\end{array}$} \\
\hline & $\overline{P^{\mathrm{V}}}$ & $V^{\mathrm{L}}$ & $\overline{p^{v}}$ & $V^{\prime}$ & & & $\begin{array}{l}\overline{\mathrm{AAD}} \\
(P)^{\circ} \%\end{array}$ & $\begin{array}{l}\text { AAD } \\
\left(v^{\prime}\right) ;\end{array}$ & \\
\hline Cyclohexanone & $322-511$ & $308-350$ & 28 & 7 & -0.03808 & 0.10161 & 0.86 & 0.13 & {$[16]$} \\
\hline Dimethylether & $177-248$ & $200-338$ & 12 & 56 & 0.14752 & 0.25491 & $1.5+$ & 0.57 & [21] \\
\hline Diethylether & $250-467$ & $239-374$ & 59 & 45 & 0.19306 & 0.23807 & 0.7 .3 & 1.05 & {$[22.23]$} \\
\hline Isopropylether & $422-494$ & $245-440$ & 14 & 60 & 0.32629 & 0.35998 & 0.39 & 0.77 & [21] \\
\hline Methylpropylether & $254-3.33$ & $245-401$ & 22 & 47 & 0.17155 & 0.27569 & 0.52 & 1.05 & {$[21.24]$} \\
\hline Isopropylmethylether & $250-.325$ & $242-374$ & 20 & 42 & 0.1856 .3 & 0.26986 & 0.4 .5 & 0.99 & {$[21.24]$} \\
\hline Dipropylether & $293-388$ & $272-431$ & 23 & 52 & 0.15101 & 0.4 .3450 & 0.41 & 1.01 & {$[21.24]$} \\
\hline Vinylethylether & $366-472$ & $269-416$ & 20 & 47 & 0.05679 & 0.71583 & 0.73 & 0.65 & [21] \\
\hline Butylmethylether & $266-367$ & $257-413$ & 25 & 49 & 0.17890 & 0.28760 & 0.52 & 0.98 & $\lceil 21.24]$ \\
\hline Ethanoic acid & $304-391$ & $293-440$ & 22 & 10 & 0.06516 & -0.078 .38 & 0.68 & 0.79 & {$[25]$} \\
\hline Propanoic acid & $308-347$ & $293-443$ & 13 & 17 & 0.06602 & -0.06286 & 1.44 & 0.92 & {$[25]$} \\
\hline Butanoic acid & $36.3-396$ & $298-483$ & 13 & 20 & 0.17767 & 0.04272 & 1.53 & $0.5 x$ & {$[25]$} \\
\hline Pentanoic acid & $377-405$ & $298-483$ & 11 & 20 & 0.17233 & 0.03134 & 1.46 & 0.76 & {$[25]$} \\
\hline Benzene acid & $4.36-523$ & $403-453$ & 6 & 6 & 0.466 .39 & 0.69242 & 0.99 & 0.08 & {$[14]$} \\
\hline Methylacetate & $260-351$ & $273-493$ & 25 & 23 & 0.18823 & 0.18493 & 1.13 & 1.12 & [26] \\
\hline Ethylacetate & $271-523$ & $27.3-473$ & 29 & 21 & 0.22821 & 0.18793 & 0.67 & 0.53 & {$[1+.26]$} \\
\hline Propylicetate & $290-399$ & $297-373$ & 28 & 10 & 0.23291 & 0.18416 & 0.75 & 0.12 & {$[26,27]$} \\
\hline Butylacetate & $326-405$ & $273-383$ & 15 & 13 & 0.22433 & 0.27146 & 0.47 & 0.89 & {$[27]$} \\
\hline Methyl formal & $294-305$ & $273-443$ & 9 & 19 & 0.06879 & 0.17135 & 1.05 & $0.6 x$ & {$[18]$} \\
\hline Ethyl format & $277-323$ & $273-463$ & 12 & 21 & 0.20279 & $0.18+77$ & 1.08 & 0.61 & {$[1 \times]$} \\
\hline Propyl format & $308-355$ & $273-513$ & 13 & 26 & 0.19834 & 0.21727 & 101 & 0.67 & {$[18]$} \\
\hline 1-Propylamine & $296-330$ & $233-431$ & 7 & 64 & 0.08544 & 0.27094 & 1.92 & 1.14 & {$[21.28]$} \\
\hline 2-Propylamine & $277-3,34$ & $230-431$ & 9 & 64 & 0.15897 & 0.31352 & 1.67 & 1.26 & {$[21.28]$} \\
\hline Trimethylamine & $221-276$ & $200-413$ & 16 & 72 & 0.13887 & 0.23705 & 1.18 & 0.91 & {$[21,28]$} \\
\hline Diethylamine & $30.5-334$ & $230-431$ & 13 & 68 & 0.39534 & $0.170 ! 7$ & 0.64 & 0.50 & [21.28] \\
\hline Water & $273-647$ & $289-578$ & 97 & 60 & 0.11560 & 0.01471 & 0.97 & 1.60 & {$[14]$} \\
\hline Grand average & & & 4182 & 3995 & & & 0.78 & 0.42 & \\
\hline
\end{tabular}

$\operatorname{AAD}(\%)=\frac{100 \mathrm{eg}}{n} \sum\left[\left|X^{\mathrm{cxp}} \cdots X^{\mathrm{cal} \mid}\right| / X^{\mathrm{cxp}}\right] ; X=P$ or $V^{\mathrm{l}}$.

Translation along the volume axis affects the saturated density calculations, and leaves vapor pressure conditions unchanged. Peneloux et al. [5] used a linear volume translation in the SRK EOS [6] and correlated the volume translation parameter as a function of the Rackett compressibility factor. This method gives satisfactory results on nonpolar systems while large errors exist for polar fluids. Yu and Lu [7] also applied the volume translation technique to the Peng-Robinson EOS and presented the translated PR (TPR) EOS. Carrier et al. [8] used a volume translated cubic EOS to hydrocarbon systems and presented the parameters in a group contribution form. Their work was further extended to high pressure calculations by Rogalski et al. [9]. Watson et al. [10] employed the volume translation concept to the VDW-711 EOS and correlated the volume translation parameter as functions of the critical constants, the acentric factor and the reduced temperature. Magoulas and Tassios [11] presented a translated modified Peng-Robinson ( $t-m P R$ ) EOS and showed obvious improvements on the liquid density calculations of alkanes. Satyro and Trebble [12] showed that the volume translation parameter for alkane molecules can be correlated as a function of their molecular weight. Very 


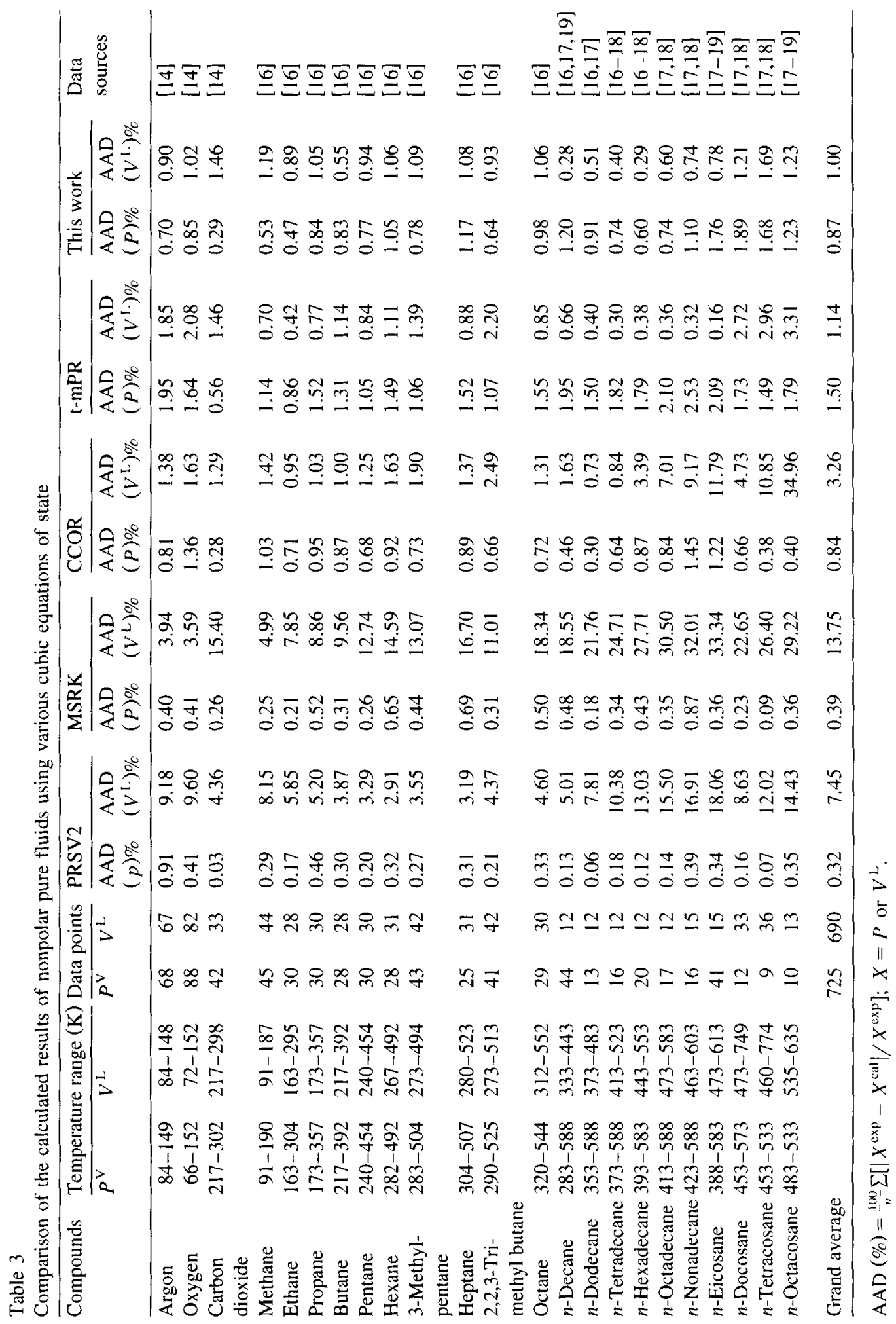




\begin{tabular}{|c|c|c|c|c|c|c|c|}
\hline$\frac{\pi}{g}$ & 苞 & 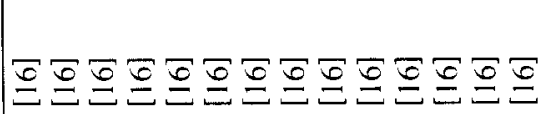 & 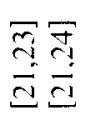 & $\overline{\bar{a}}$ & 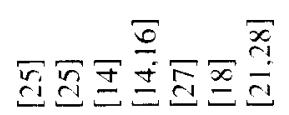 & 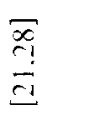 & \\
\hline 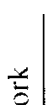 & 这吉 & 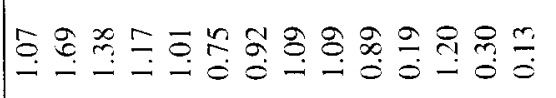 & $\stackrel{2}{\circ}$ & 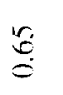 & 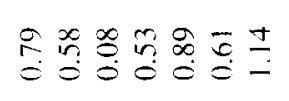 & $\stackrel{\widetilde{s}}{=}$ & $\stackrel{m}{s}$ \\
\hline & $\frac{8}{2}$ & 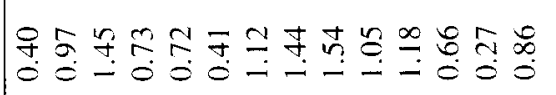 & $\stackrel{m}{\stackrel{n}{+}}$ & $\stackrel{\check{1}}{\Xi}$ & 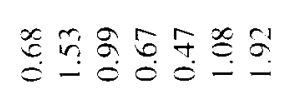 & $\stackrel{t}{\Xi}$ & $\stackrel{\ddot{x}}{\stackrel{x}{\infty}}$ \\
\hline & $\sum_{i}^{2}$ & 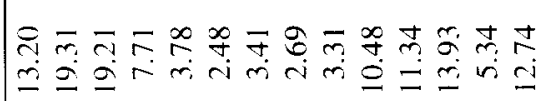 & 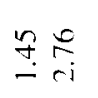 & $\begin{array}{l}\frac{r}{2} \\
\stackrel{2}{2}\end{array}$ & 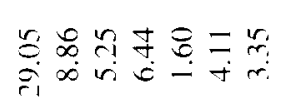 & $\underset{r i}{v}$ & 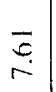 \\
\hline $\begin{array}{l}2 \\
2 \\
2 \\
2 \\
2\end{array}$ & $\sum_{2}^{2}$ & 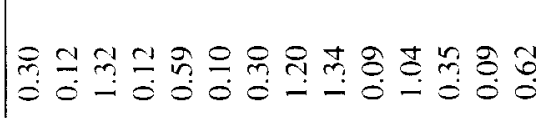 & $\begin{array}{l}\infty \\
\stackrel{\infty}{0} \\
\vdots\end{array}$ & $\stackrel{\theta}{G}$ & 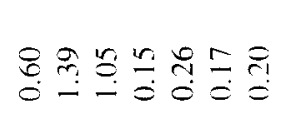 & sis & $\stackrel{\substack{c \\
\stackrel{+}{=}}}{=}$ \\
\hline & 是突 & 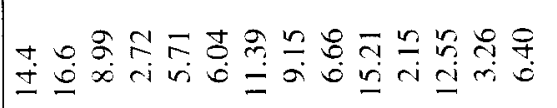 & 象落 & $=$ & 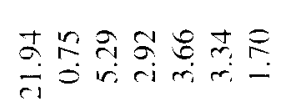 & $\begin{array}{l}\bar{x} \\
r i\end{array}$ & 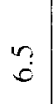 \\
\hline & $\frac{0}{2}$ & 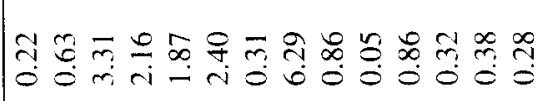 & $\stackrel{\infty}{\stackrel{\infty}{\circ}}$ & $\underset{\Xi}{\exists}$ & 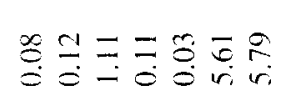 & $\frac{1}{3}$ & $\underline{m}$ \\
\hline & $\mid \frac{2}{2}$ & 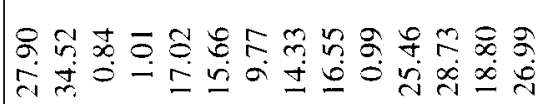 & $\begin{array}{l}\Xi \\
\stackrel{\Xi}{\Xi}\end{array}$ & $\begin{array}{l}\infty \\
\stackrel{\infty}{0} \\
+\end{array}$ & 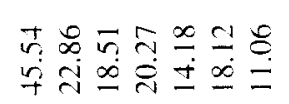 & $\overline{m_{i}}$ & 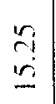 \\
\hline$\left|\begin{array}{l}\frac{1}{2} \\
\frac{\alpha}{2} \\
\frac{0}{2}\end{array}\right|$ & $\frac{25}{2}$ & 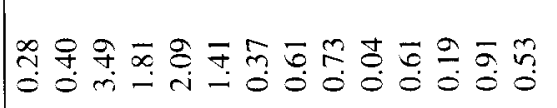 & $\stackrel{+}{\stackrel{+}{=}}$ & $=$ & 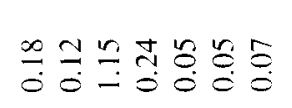 & $\Xi_{5}$ & $\stackrel{\Xi}{\check{\sigma}}$ \\
\hline & $\mid \begin{array}{l}2 \\
\frac{2}{2}\end{array}$ & 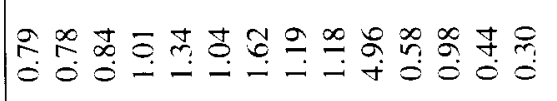 & 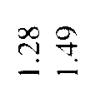 & $\begin{array}{l}\Xi \\
\Xi\end{array}$ & 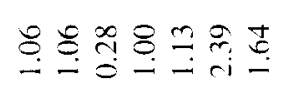 & $\stackrel{+}{\infty}$ & $\hat{G}$ \\
\hline 5 & $\mid \frac{2}{3} \frac{2}{2}$ & 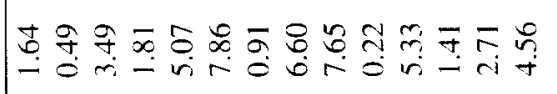 & 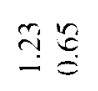 & $\stackrel{n}{\infty}$ & 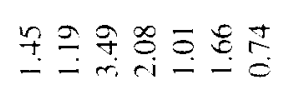 & $\stackrel{?}{+}$ & $\underset{i}{+}$ \\
\hline 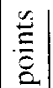 & $\dot{\Delta}$ & 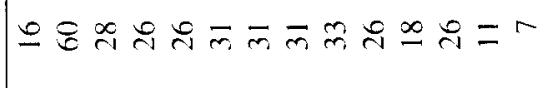 & 舟 & 守 & 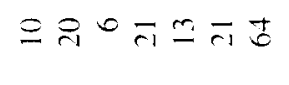 & $\mathscr{x}$ & $\hat{n}$ \\
\hline $\mid$ & 12 & त̄ & a $\approx$ & 8 & ind & $\stackrel{\sim}{-}$ & $\underset{D}{Z}$ \\
\hline 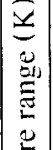 & $\dot{S}$ & 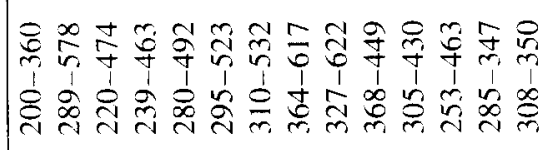 & 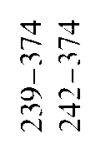 & 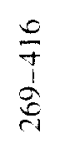 & 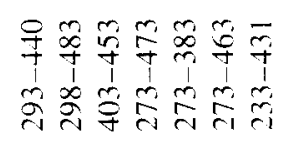 & 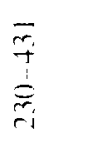 & \\
\hline 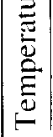 & 定 & 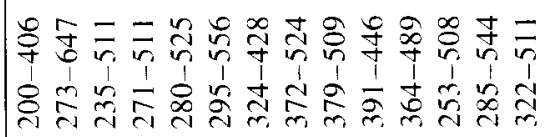 & 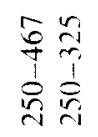 & 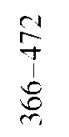 & 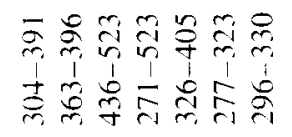 & 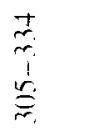 & \\
\hline 莺 & & 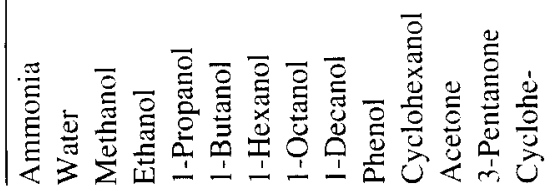 & $\sum_{\overline{ \pm}}^{\bar{\Xi}} \bar{Z}$ & $\sum_{\bar{L}}^{\frac{2}{U}}$ & 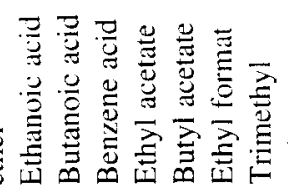 & 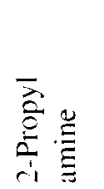 & 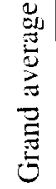 \\
\hline
\end{tabular}




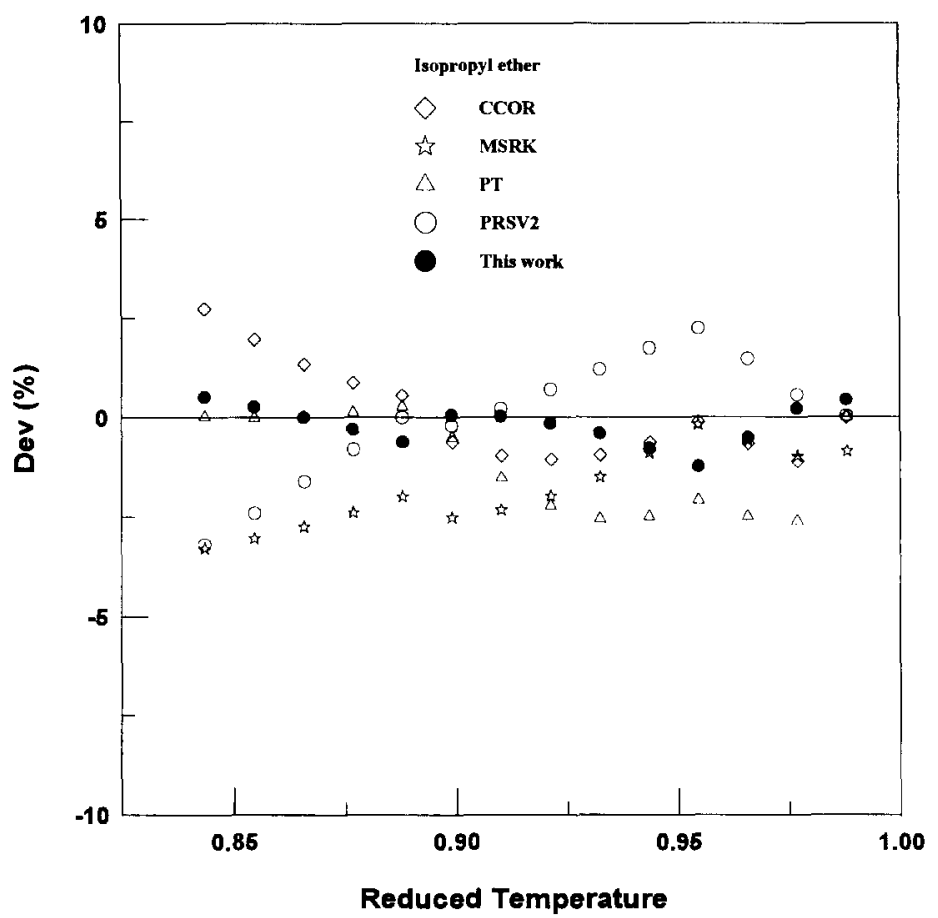

Fig. 1. Error distributions of the calculated saturated vapor pressures of isopropyl ether by various equations of state.

recently, Kenney et al. [13] developed a hard-sphere volume translated van der Waals EOS to model the supercritical water oxidation process. It is observed that the volume translation technique yields improved volume calculation results and previous investigations are usually limited to the nonpolar hydrocarbon systems. In this study, we apply the volume translated Peng-Robinson EOS to both nonpolar and polar fluids. Optimal pure fluid parameters are presented and their volumetric and phase equilibrium calculations are compared with those from other EOS methods.

\subsection{Development of the VTPR EOS}

Applying the volume translation technique, the VTPR EOS is written as:

$$
P=\frac{R T}{V+t-b}-\frac{a}{(V+t)(V+t+b)+b(V+t-b)}
$$

where $t$ is the translated volume parameter with which the volume calculated by this EOS approaches the experimental value:

$$
V_{\text {exp }}=V_{\text {EOS }}+t
$$

The EOS parameters $a$ and $b$ are determined by the critical properties:

$$
\left(\frac{\partial P}{\partial V}\right)_{c}=\left(\frac{\partial^{2} P}{\partial V^{2}}\right)_{c}=0
$$




$$
\begin{aligned}
& a=0.45724 \frac{R^{2} T_{c}^{2}}{P_{c}} \alpha(T) \\
& b=0.07780 \frac{R T_{c}}{P_{c}}
\end{aligned}
$$

Various expressions of $\alpha$ had been presented in literature. In this study, it is also correlated as a function of temperature. To determine the pure fluid parameters, the equal fugacity criterion was employed:

$$
f^{\prime}=f^{\mathrm{L}}
$$

The fugacity coefficient of the VTPR EOS is:

$$
\ln \phi=(Z+T *-1)-\ln (Z+T *-B)-\frac{A}{2 \sqrt{2} B} \ln \left[\frac{Z+T *+(1+\sqrt{2}) B}{Z+T *+(1-\sqrt{2}) B}\right]
$$

where

$$
\begin{aligned}
& A=\frac{P a}{R^{2} T^{2}} \\
& B=\frac{P b}{R T} \\
& T *=\frac{P t}{R T}
\end{aligned}
$$

Regressions of the saturated properties of pure fluids were carried out along the saturation curve with the following objective function:

$$
\mathrm{OF}=\frac{100}{n} \sum_{i}^{n} \frac{\left|P_{i}^{\exp }-P_{i}^{\text {cal }}\right|}{P_{i}^{\exp }}+\frac{100}{n} \sum_{i}^{n} \frac{\left|V_{i}^{\exp }-V_{i}^{\text {cal }}\right|}{V_{i}^{\exp }}
$$

The VTPR EOS was also employed in VLE calculations where the following mixing rules were used:

$$
\begin{aligned}
& a_{\mathrm{m}}=\sum_{i} \sum_{j} x_{i} x_{i} a_{i j} \\
& a_{i j}=\sqrt{a_{i} a_{j}}\left(1-k_{i j}\right) \\
& b_{\mathrm{m}}=\sum_{i} x_{i} b_{i} \\
& t_{\mathrm{m}}=\sum_{i} x_{i} t_{i}
\end{aligned}
$$




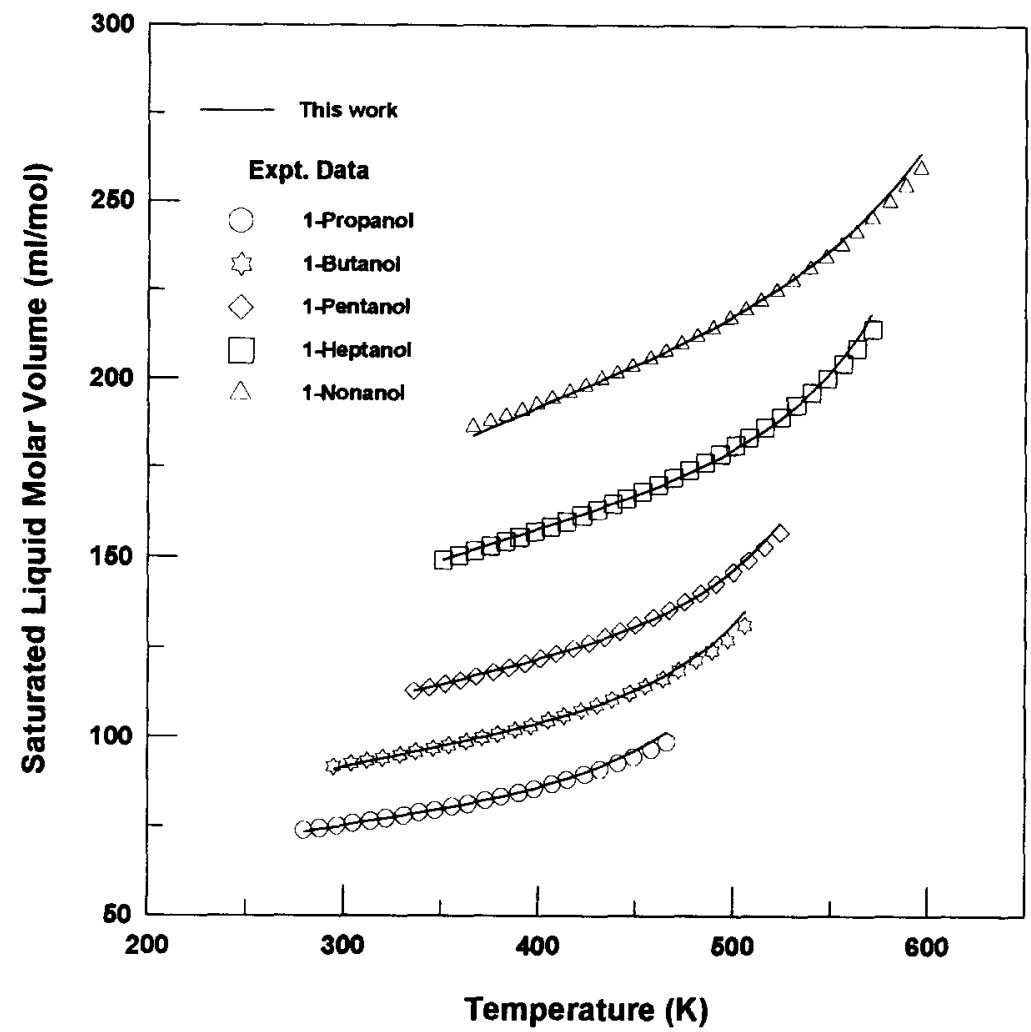

Fig. 2. Comparison of the calculated saturated liquid molar volumes of selected alcohols with experimental data [16].

The fugacity coefficient of component $i$ in a mixture is expressed as:

$$
\ln \bar{\phi}_{i}=\frac{b_{i}}{B}(Z+\bar{t}-1)-\ln (Z+\bar{t}-1)-\frac{A}{2 \sqrt{2 B}}\left[\frac{2 \sum_{j} x_{j} a_{i j}}{A}-\frac{b_{i}}{B}\right] \ln \left[\frac{Z+\bar{t}+(1+\sqrt{2}) B}{Z+\bar{t}+(1-\sqrt{2}) B}\right]
$$

$A$ and $B$ are defined as shown in Eqs. (8) and (9) for a fluid mixture and

$$
\bar{t}=\frac{P t_{\mathrm{m}}}{R T}
$$

One binary interaction parameter $k_{\mathrm{ij}}$ was used in VLE calculations of fluid mixtures. The optimal values of $k_{\mathrm{ij}}$ were obtained using the following objective function:

$$
\mathrm{OF}^{\prime}=\frac{100}{n} \sum_{i}^{n}\left[\left|\frac{P_{i}^{\text {cal }}-P_{i}^{\text {exp }}}{P_{i}^{\text {exp }}}\right|+\left|y_{i}^{\text {cal }}-y_{i}^{\exp }\right|\right]
$$




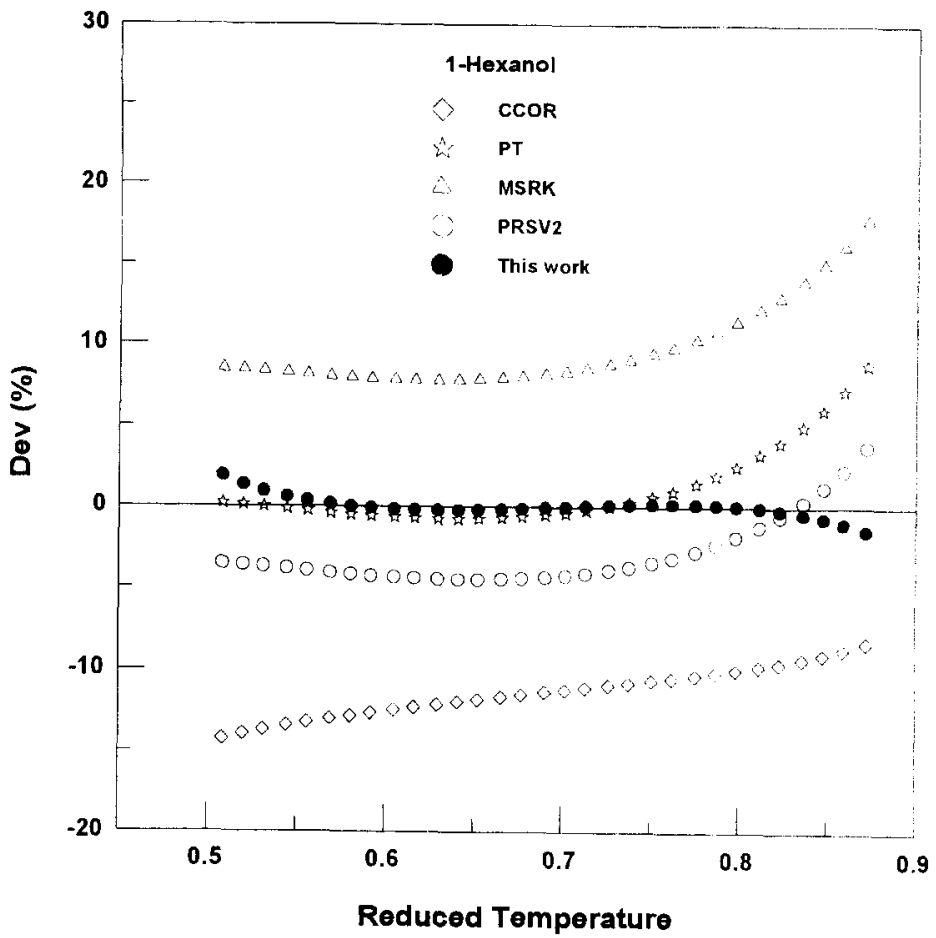

Fig. 3. Comparison of the calculated saturated liquid molar volumes of 1-hexanol from various equations of state. Data source: Ref. [16].

Table 5

Comparison of saturated vapor molar volumes of pure fluids using various cubic equations of state

\begin{tabular}{lccccccc}
\hline Compounds & Temperature range $(K)$ & Data points & \multicolumn{2}{c}{ AAD $\left(V^{\vee}\right) \%$} \\
\cline { 4 - 7 } & & & \multicolumn{1}{c}{ PT } & MSRK & CCOR & PRSV2 & This work \\
\hline Methane & $91-176$ & 39 & 1.23 & 0.49 & 6.68 & 0.89 & 0.96 \\
Octane & $312-504$ & 25 & 1.63 & 1.44 & 4.29 & 1.67 & 1.26 \\
Benzene & $284-517$ & 37 & 1.74 & 1.10 & 4.41 & 2.30 & 1.14 \\
Chloromethane & $193-372$ & 35 & 1.60 & 3.61 & 3.39 & 1.48 & 1.48 \\
Sulfur dioxide & $323-378$ & 12 & 3.00 & 2.44 & 6.38 & 1.85 & 1.87 \\
Ammonia & $200-406$ & 13 & 2.93 & 2.88 & 1.59 & 4.02 & 1.86 \\
Methanol & $227-459$ & 32 & 5.20 & 3.55 & 9.41 & 12.62 & 1.48 \\
Ethanol & $231-423$ & 20 & 2.14 & 5.28 & 3.86 & 2.31 & 1.49 \\
I-Propanol & $280-475$ & 24 & 5.72 & 3.52 & 5.16 & 7.94 & 1.18 \\
l-Butanol & $295-506$ & 26 & 7.92 & 2.71 & 5.42 & 9.80 & 1.09 \\
1-Nonanol & $366-440$ & 10 & 40.63 & 47.30 & 4.54 & 0.84 & 0.82 \\
Acetone & $253-335$ & 12 & 2.98 & 1.68 & 0.38 & 3.00 & 1.60 \\
Water & $273-598$ & 80 & 2.15 & 1.72 & 4.48 & 9.54 & 1.59 \\
Grand average & & 365 & 3.97 & 3.54 & 4.93 & 5.45 & 1.36 \\
\hline
\end{tabular}

Data source: Ref. [16]

$\operatorname{AAD}\left(V^{v}\right) \%=\frac{100}{n} \Sigma\left[\left|V^{\vee \exp }-V^{\vee \text { cal }}\right| / V^{v \exp }\right]$. 


\section{Results and discussion}

The VTPR EOS has been used to correlate the saturated properties of pure nonpolar and polar fluids. A new temperature-dependent function of $\alpha$ is obtained:

$$
\alpha(T)=\left[1+M\left(1-T_{\mathrm{R}}\right)+N\left(1-T_{\mathrm{R}}\right)\left(0.7-T_{\mathrm{R}}\right)\right]^{2}
$$

where $M$ and $N$ are two parameters for each pure fluid. It is found that $M$ can be correlated as a function of the acentric factor:

$$
M=0.20473+0.83548 \omega-0.18470 \omega^{2}+0.16675 \omega^{3}-0.09881 \omega^{4}
$$

No suitable correlation for $N$ was obtained and it is left as a pure fluid parameter for the VTPR EOS. The translated volume parameter $t$ has been examined for various pure fluids. At reduced temperatures less than 0.8 , a constant value of $t$ is simple enough to result in satisfactory improvement of the calculated saturated liquid molar volumes. At higher reduced temperatures, $t$ is better treated as a temperature-dependent parameter. In this study, $t$ is taken as the following temperature-dependent form in correlating the saturated properties:

$$
t=\frac{R T_{\mathrm{c}}}{P_{\mathrm{c}}}\left[k_{\mathrm{1}}+k_{2}\left(1-T_{\mathrm{R}}^{2 / 3}\right)+k_{3}\left(1-T_{\mathrm{R}}^{2 / 3}\right)^{2}\right]
$$

Further regression of the $k$ parameters in the above equation has been investigated. It is found that $k_{1}$

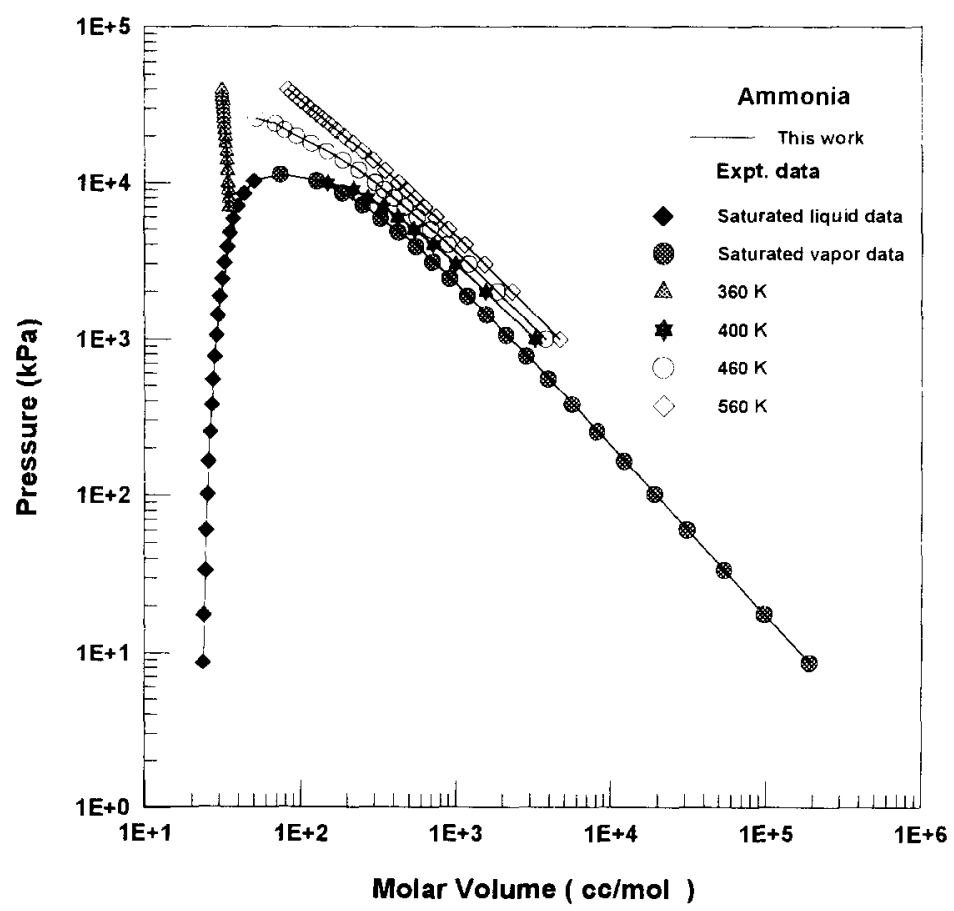

Fig. 4. Comparison of the calculated $P V T$ data from the VTPR EOS for ammonia with experimental data [14]. 


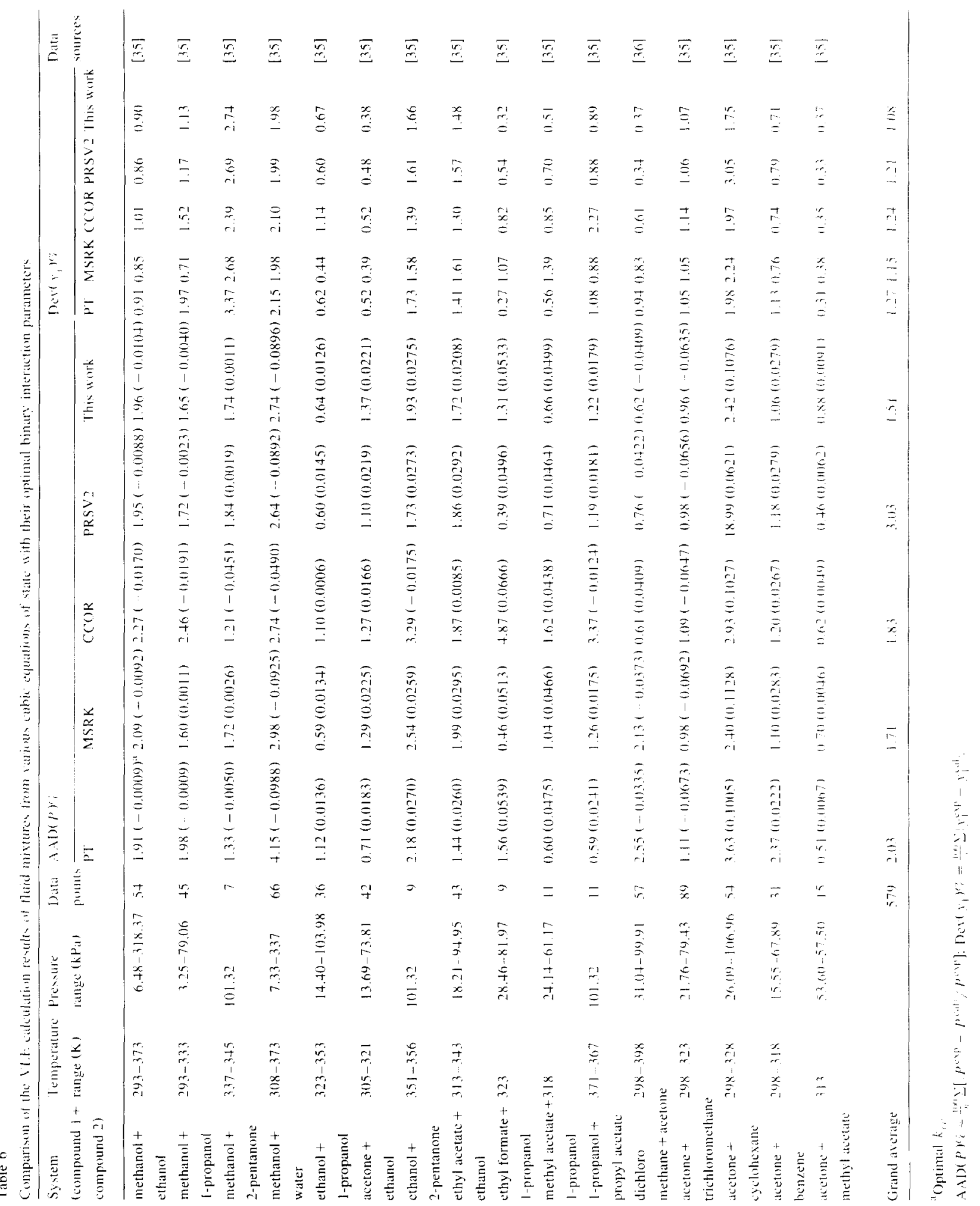




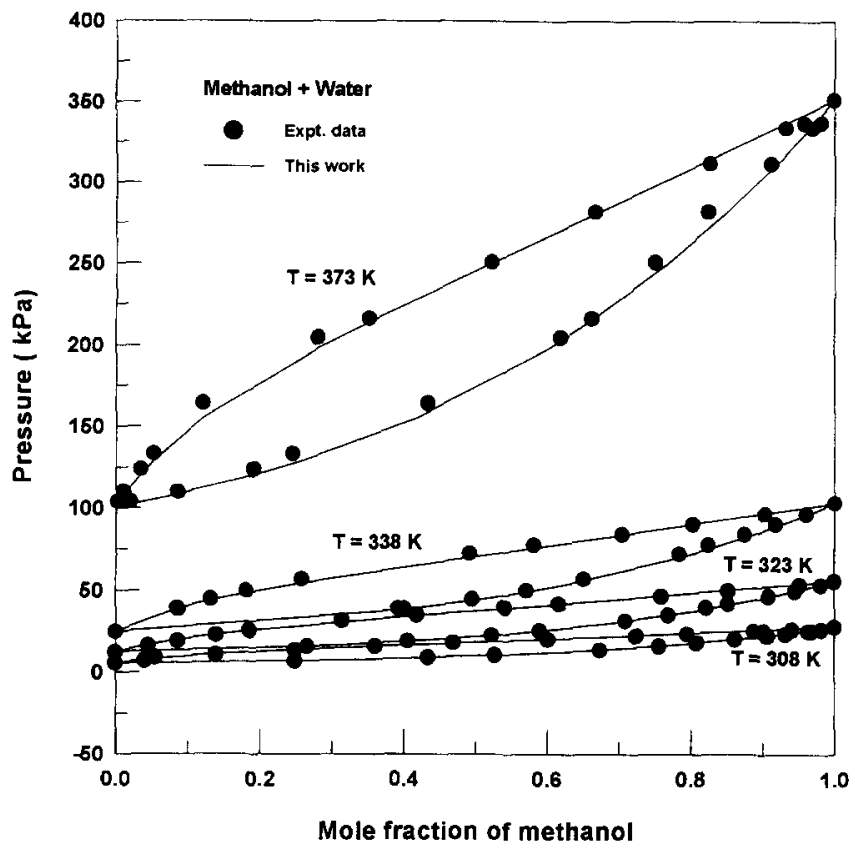

Fig. 5. VLE calculation results from the VTPR EOS and the VDW mixing rules for methanol and water binary mixtures at various temperatures $\left(k_{i j}=-0.0896\right)$. Data source: Ref. [35].

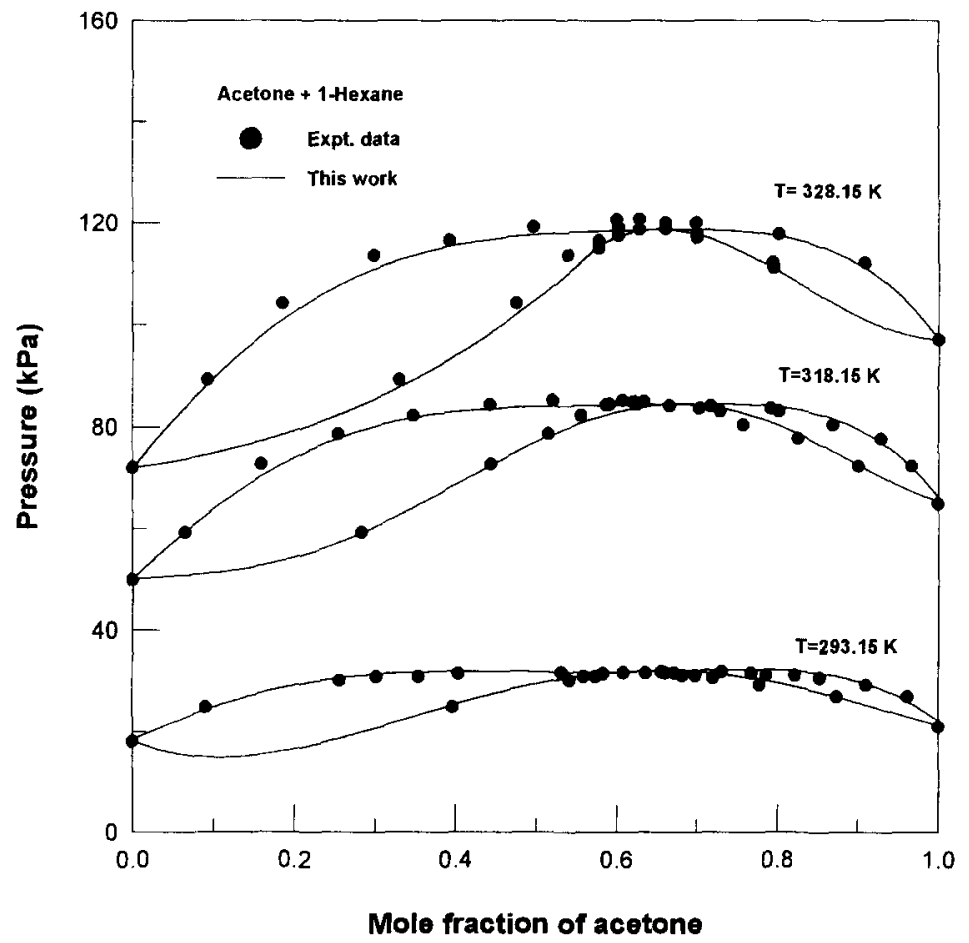

Fig. 6. VLE calculation results from the binary mixtures of acetone and 1-hexane at various temperatures using the VTPR EOS with MHV2 mixing rules. Data source: Ref. [35]. 
can be expressed as a function of the acentric factor, $k_{3}$ is left as another pure fluid parameter, and $k_{2}$ can be regressed as a function of $k_{3}$ :

$$
\begin{aligned}
& k_{1}=0.00185+0.00438 \omega+0.36322 \omega^{2}-0.90831 \omega^{3}+0.55885 \omega^{4} \\
& k_{2}=-0.00542-0.51112 k_{3}+0.04533 k_{3}^{2}+0.07447 k_{3}^{3}-0.03831 k_{3}^{4}
\end{aligned}
$$

Thus the VTPR EOS has two pure fluid parameters $N$ and $k_{3}$, in addition to the critical properties and acentric factors. These pure fluid parameters have been regressed for over 130 pure components and their values are listed in Tables 1 and 2. The overall absolute average deviations in vapor pressure and saturated liquid molar volume are less than 1\%. The VTPR EOS has been compared with other cubic EOS (PRSV2 [1,2]; MSRK [29]; PT [30]; CCOR [31,32]; t-mPR [11]). The PRSV2 EOS has three parameters, and each other EOS has two parameters for every pure fluid. The pure fluid parameters of various EOS have been refitted using the same pure fluid data base in the comparisons. The results for saturated property calculations are listed in Tables 3 and 4 for nonpolar and polar pure fluids, respectively. The PRSV2, MSRK, and the CCOR EOS give accurate results for vapor pressures, but relatively larger deviations in saturated liquid molar volumes. The $t$-mPR EOS yields improved liquid molar volume calculations for nonpolar fluids. The generalized correlations for the nonpolar fluid parameters of the t-mPR EOS can not yield satisfactory results for polar systems. For the polar fluids. it is observed that the PT EOS gives good results on saturated liquid molar volumes, but larger deviations in vapor pressures. The VTPR EOS developed in this study shows satisfactory results for both nonpolar and polar fluids. The absolute average deviations in vapor pressure and saturated liquid molar volume are less than $1 \%$, and the peak deviations are less than $1.5 \%$. Fig. 1 shows an example

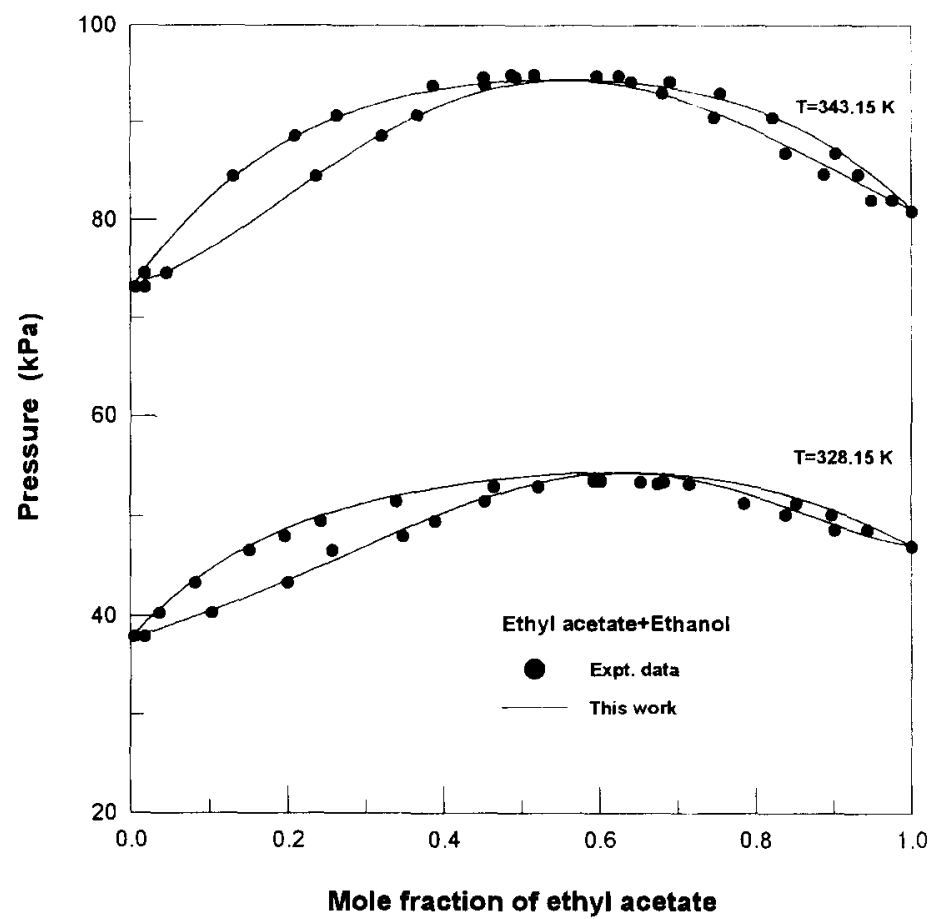

Fig. 7. VLE calculation results from the binary mixtures of ethyl acetone and ethanol at various temperatures using the VTPR EOS with LCVM mixing rules. Data source: Ref. [35] 


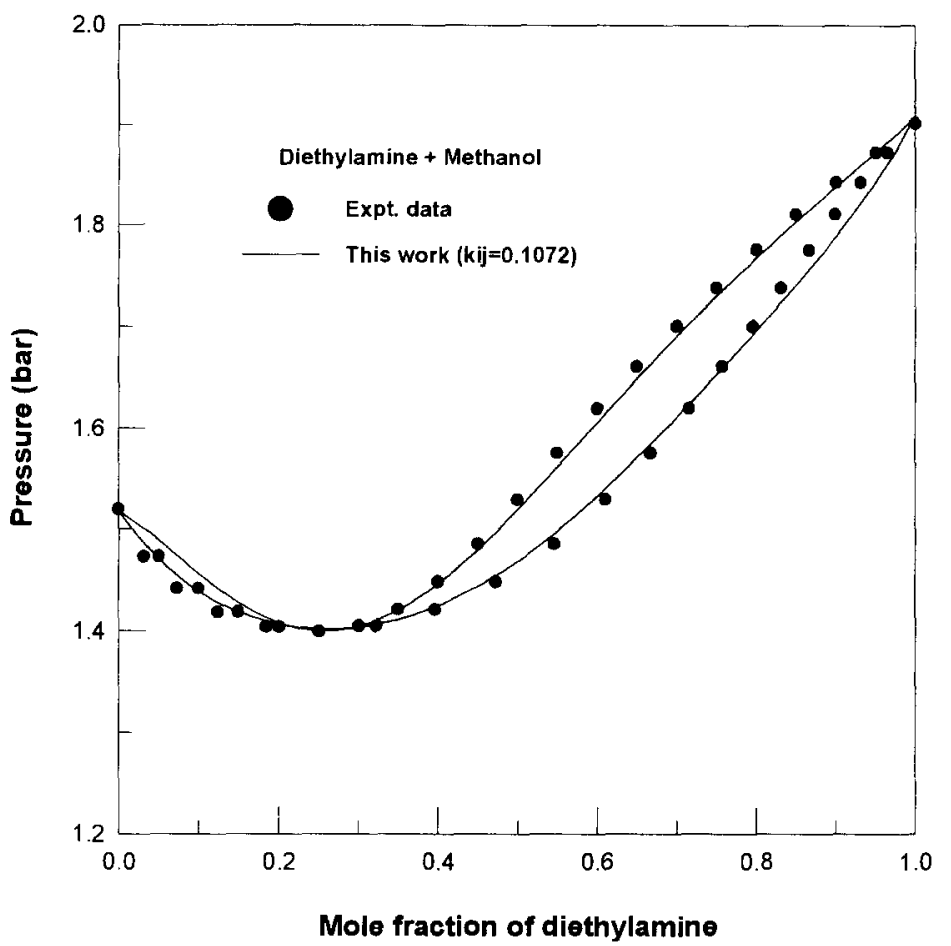

Fig. 8. VLE calculation results from the binary mixtures of diethylamine and methanol at $348.09 \mathrm{~K}$ using the VTPR EOS with Wong-Sandler mixing rules. Data source: Ref. [42].

of the error distributions of the vapor pressure calculations of isopropyl ether from various EOS. The VTPR EOS is satisfactory in a wide range of reduced temperature. Fig. 2 shows the calculated results of the saturated liquid molar volumes of alcohols from the VTPR EOS. Good agreement with the experimental data is observed up to the reduced temperatures higher than 0.9. Fig. 3 shows a graphical comparison of the error distribution on the saturated liquid molar volume calculations of 1-hexanol using various EOS. The VTPR EOS gives general good results and the smallest deviation at higher reduced temperature range. The VTPR EOS also yields satisfactory results on the saturated vapor volumes. A comparison of the calculation results with other EOS models is shown in Table 5. It is presented that the grand average absolute deviation from the VTPR EOS is again superior to other EOS. A comparison of the calculated $P V T$ data of ammonia is shown in Fig. 4, and the agreement with experimental data is satisfactory. Very recently, we have developed a three-parameter cubic perturbed hard body (CPHB) EOS [33], and have shown satisfactory results for nonpolar and polar fluids. Both the CPHB and VTPR EOS show good results on vapor pressures with absolute average deviation less than $1 \%$ for nonpolar and polar pure fluids. The VTPR EOS, with less pure fluid parameters, shows further improvement on liquid volume results than those from the CPHB EOS. Both EOS parameters are obtained by regressing the saturated properties of pure fluids. They may have limitations for direct application to supercritical conditions. For certain compounds without experimental saturated property data, values estimated from correlation equations [34] can be used to regress the EOS parameters. In this case, the predicted results depend on the accuracy of the correlation equations. 


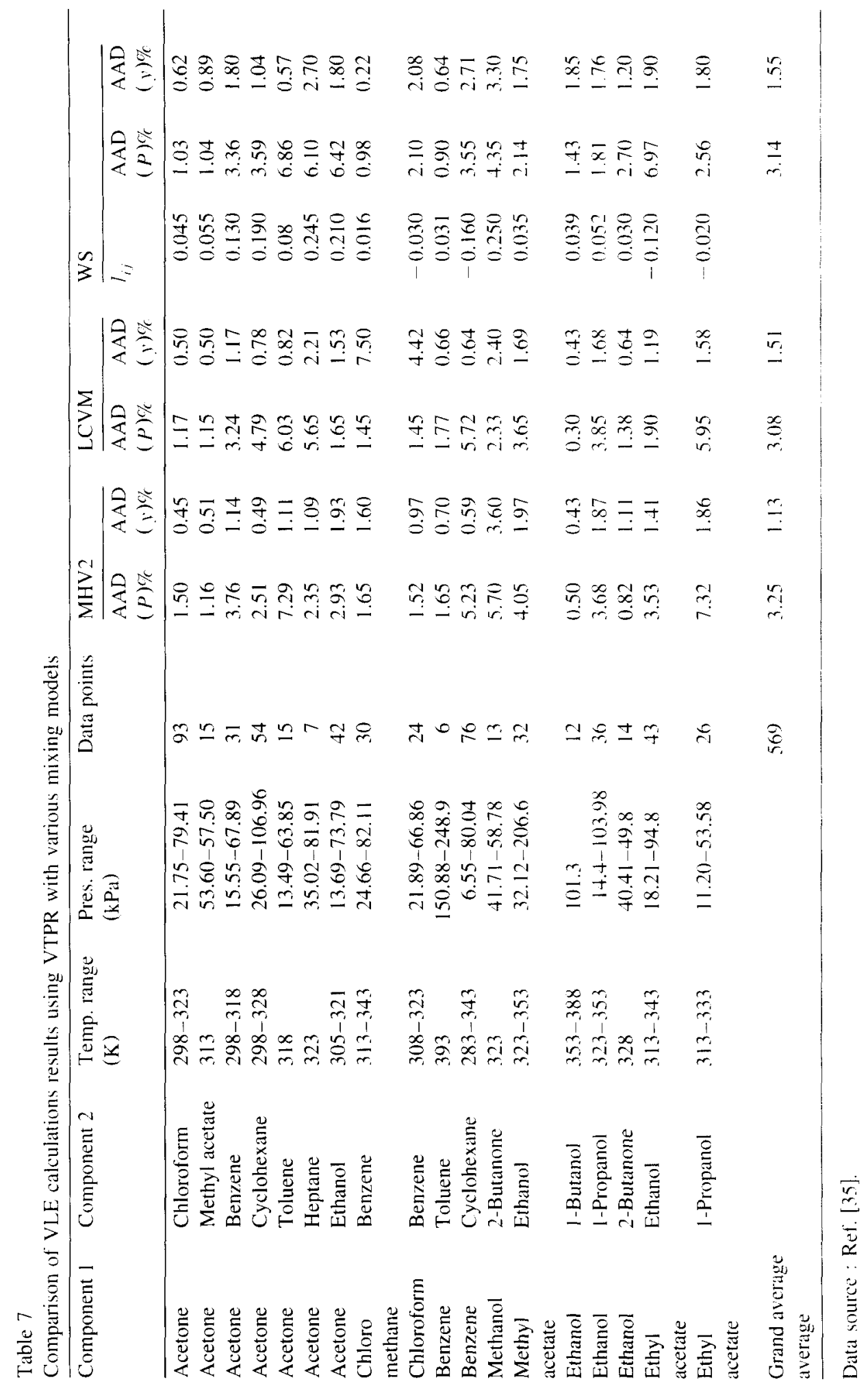


Table 8

Comparison of liquid density calculations of fluid mixtures at atmospheric pressure using various cubic equations of state

\begin{tabular}{|c|c|c|c|c|c|c|c|c|}
\hline \multirow[t]{2}{*}{ System } & \multirow[t]{2}{*}{ Temperature (K) } & \multirow[t]{2}{*}{ Data points } & \multicolumn{5}{|c|}{$\operatorname{AAD}\left(\rho^{\mathrm{L}}\right) \%$} & \multirow[t]{2}{*}{ Data sources } \\
\hline & & & MSRK & CCOR & PRSV2 & PT & This work & \\
\hline$n$-hexane $+n$-decane & 298.15 & 9 & 9.92 & 0.574 & 2.41 & 1.00 & 0.22 & [43] \\
\hline$n$-octane $+n$-decane & 298.15 & 9 & 11.23 & 0.89 & 3.75 & 1.39 & 0.52 & [43] \\
\hline$n$-nonane $+n$-decane & 298.15 & 9 & 11.77 & 1.21 & 4.28 & 1.69 & 0.73 & [43] \\
\hline toluene + octane & 298.15 & 10 & 9.42 & 0.78 & 1.39 & 0.62 & 0.56 & [44] \\
\hline toluene $+n$-decane & 303.15 & 10 & 10.68 & 0.59 & 2.62 & 1.14 & 0.42 & [44] \\
\hline 1-hexane +1 -hexanol & 298.15 & 11 & 6.29 & 6.47 & 2.14 & 0.41 & 0.25 & [45] \\
\hline octane +1 -hexanol & 298.15 & 11 & 8.49 & 5.34 & 1.23 & 0.50 & 0.41 & [46] \\
\hline octane +1 -octanol & 298.15 & 11 & 9.72 & 4.60 & 1.64 & 1.27 & 0.96 & [46] \\
\hline water + methanol & 298.15 & 19 & 23.24 & 9.26 & 14.97 & 1.26 & 0.98 & {$[47]$} \\
\hline water + ethanol & 298.15 & 19 & 18.75 & 3.57 & 10.11 & 1.91 & 1.93 & {$[47]$} \\
\hline 1-propanol + water & 303.15 & 10 & 17.50 & 4.71 & 8.53 & 1.66 & 1.57 & [48] \\
\hline acetone + water & 298.15 & 14 & 21.21 & 11.02 & 12.89 & 2.21 & 1.79 & {$[48]$} \\
\hline methanol + ethanol & 323.15 & 19 & 15.78 & 2.56 & 7.96 & 0.79 & 0.67 & {$[48]$} \\
\hline 1-octanol + methanol & 298.15 & 19 & 13.37 & 5.24 & 5.05 & 2.34 & 0.36 & [47] \\
\hline 1-octanol + ethanol & 298.15 & 19 & 11.36 & 3.06 & 2.83 & 2.05 & 0.38 & [47] \\
\hline Grand Average & & 199 & 14.1 & 4.37 & 6.19 & 1.43 & 0.82 & \\
\hline
\end{tabular}

$\operatorname{AAD}\left(\rho^{\mathrm{L}}\right) \%=\frac{100}{n} \Sigma\left[\left|\rho^{\mathrm{L} \exp }-\rho^{\mathrm{L} \text { cal }}\right| / \rho^{\mathrm{L} \exp }\right]$

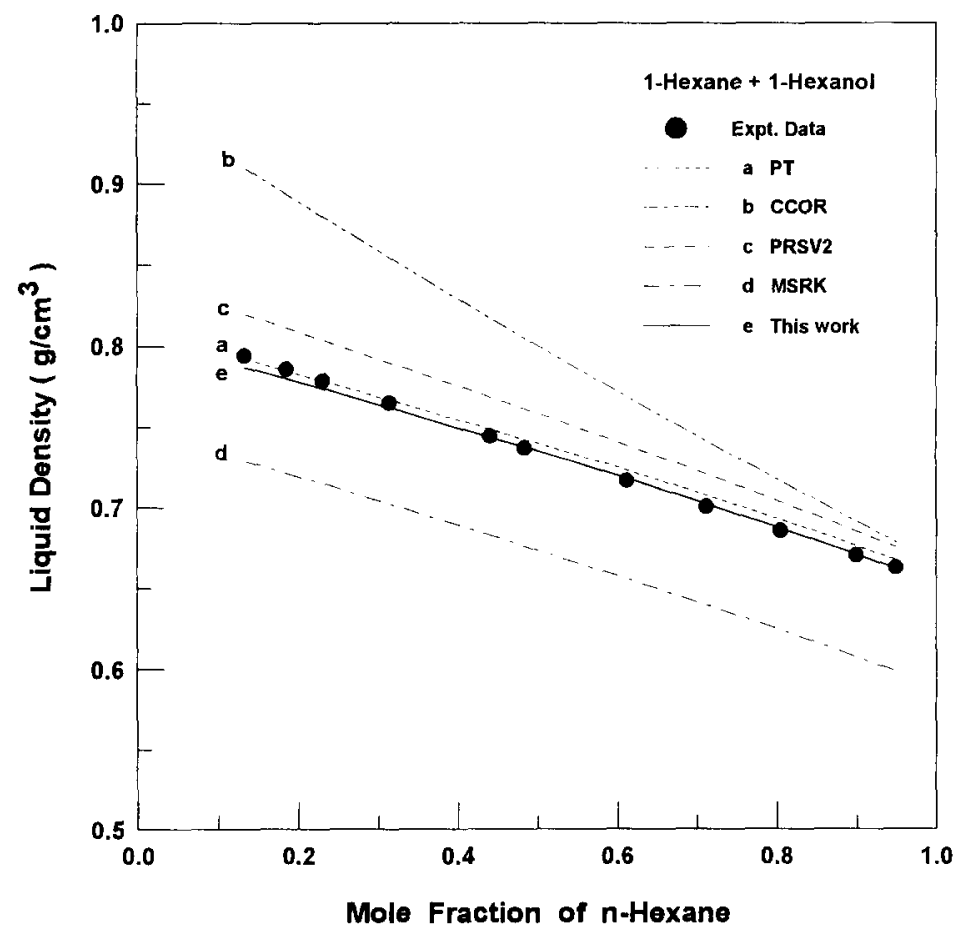

Fig. 9. Comparison of the calculated liquid densities from various equations of state for binary mixtures of 1-hexane +1 hexanol at $298.15 \mathrm{~K}$ and $101.3 \mathrm{kPa}$. Data source: Ref. [45]. 
The VTPR EOS has been employed on the VLE calculations of binary mixtures. The calculated results using traditional van der Waals one-fluid mixing rules and the comparison with other EOS are presented in Table 6. One optimally fitted binary interaction factor was used for the energy parameter of each EOS. It is shown that all EOS models give satisfactory VLE calculation results, except for certain mixtures as alcohol + normal hydrocarbon systems, with their optimally fitted parameters. A graphical presentation for the VLE calculation results using the VTPR EOS and the van der Waals mixing rules is shown in Fig. 5 for the binary mixture of methanol + water. Satisfactory agreement with the experimental data has been obtained with single temperature-independent binary interaction parameter. The VTPR EOS has also been combined with the excess free energy models in the VLE calculations. In these calculations, a group contribution UNIFAC model [37] was employed and no empirical parameter was included in the MHV2 [38,39] and LCVM [40] mixing rules. Examples are shown in Figs. 6 and 7 for the binary systems of acetone +1 -hexane and ethyl acetate + ethanol where the MHV2 and the LCVM models are employed, respectively. It is shown that the VTPR EOS yields good results for these systems from either group contribution mixing model. The azeotropic behavior can be correctly determined as shown in these figures. The Wong-Sandler mixing rules [41] were also used in the VTPR EOS, where one empirical binary interaction parameter $l_{i j}$ was included in the second virial coefficient of the mixture. Fig. 8 shows an example for the VLE calculation results from the VTPR EOS and the Wong-Sandler mixing rules for the diethylamine + methanol binary system. It is again demonstrated that the VTPR EOS with the Wong-Sandler mixing rules yields good results for this nonideal mixture with one empirical parameter. VLE calculation results using VTPR EOS + GE mixing rules are also presented in Table 7 for more binary mixtures.

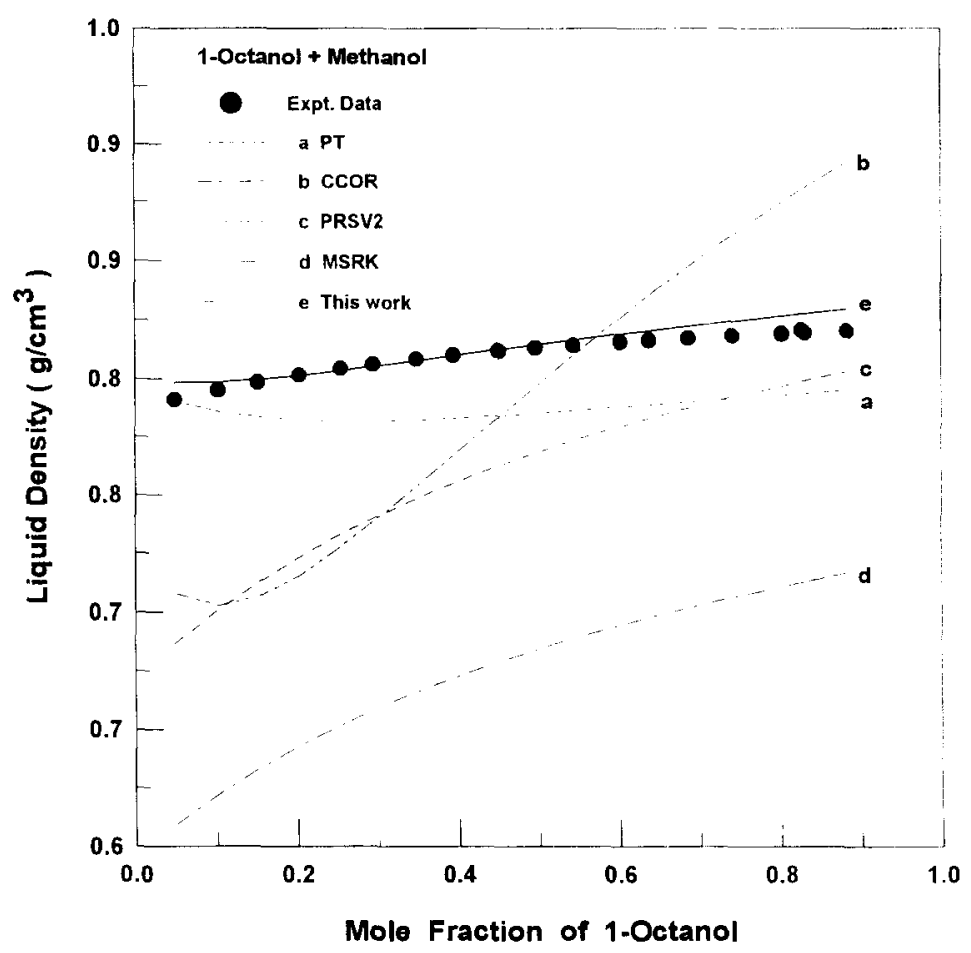

Fig. 10. Comparison of the calculated liquid densities from various equations of state for binary mixtures of 1 -octanol + methanol at $298.15 \mathrm{~K}$ and $101.3 \mathrm{kPa}$. Data source: Ref. [47]. 


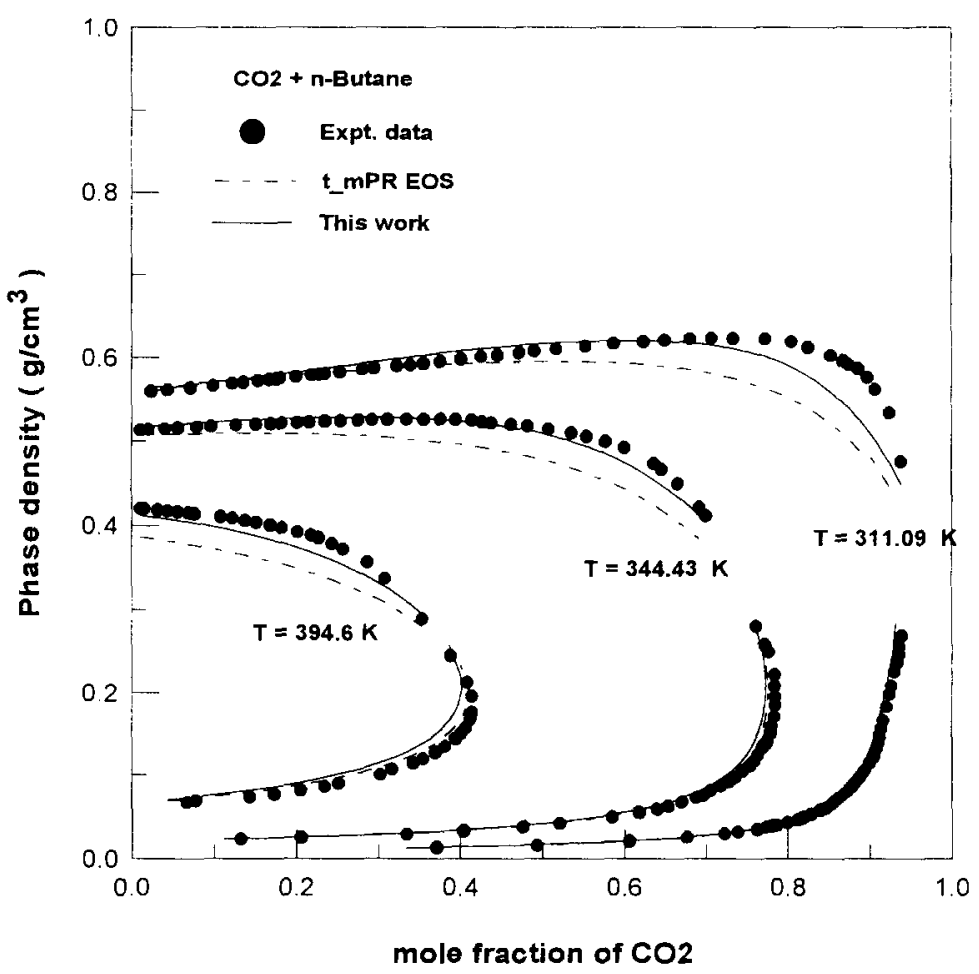

Fig. 11. Comparison of saturated densities for carbon dixoxide and $n$-butane binary mixtures calculated from t-mPR and VTPR EOS. Data source: Ref. [50].

The volume translated EOS gives improved calculated results on the liquid densities of binary mixtures at atmospheric pressure, as shown in Table 8. Five EOS models are compared for the liquid density calculations of various fluid mixtures. No binary interaction parameter was used in these calculations and all EOS were compared at a predictive basis. It is observed that the VTPR EOS yields the best accuracy. Graphical comparisons are shown in Figs. 9 and 10 for the binary mixture of 1-hexane + 1-hexanol and 1-octanol + ethanol, respectively. Generally, the PT and VTPR EOS give good agreement with the experimental data, where the VTPR EOS has the best overall accuracy. The VTPR EOS has been tested in calculating the saturated vapor and liquid molar volumes at high pressures for several binary mixtures of carbon dioxide + alkanes. Comparing to those calculated from the t-mPR EOS [49], the VTPR EOS has slightly better saturated liquid molar volume and better saturated vapor molar volume results. The calculated results for $\mathrm{CO}_{2}+n$-butane are shown in Fig. 11 at various temperatures. The pressure range in this calculation is from 5 to 80 bars. The VTPR EOS gives satisfactory results where the absolute average deviations in saturated vapor and liquid molar volumes are less than $2 \%$. It is also demonstrated that the VTPR EOS presents improvement in the saturated molar volume calculations than the original PR EOS without volume translation.

\section{Conclusion}

A volume translated Peng-Robinson (VTPR) EOS is developed in this study. Pure fluid parameters are presented over 130 nonpolar and polar compounds. The VTPR EOS shows satisfactory calculated 
results of both the vapor pressures and the saturated liquid and vapor molar volumes. VLE calculations are satisfactorily carried out using the VTPR EOS and various mixing rules. The VTPR EOS gives comparable VLE calculation results to other cubic type EOS models. The VTPR EOS. however, shows superior results on the liquid density calculations of fluid mixtures.

\section{List of symbols}

\begin{tabular}{|c|c|}
\hline$a$ & equation of state energy parameter \\
\hline$b$ & equation of state excluded volume parameter \\
\hline$f$ & fugacity for pure component $i$ \\
\hline$k_{i j}, l_{i j}$ & binary interaction parameters \\
\hline$k_{1}, k_{2}, k_{3}$ & parameters in Eq. (21) \\
\hline$n$ & number of data points \\
\hline$N, M$ & parameters in Eq. (19) \\
\hline$P$ & pressure \\
\hline$R$ & gas constant \\
\hline$t$ & volume-translation parameter \\
\hline$T$ & temperature \\
\hline$V$ & molar volume \\
\hline$x$ & mole fraction in the liquid phase \\
\hline$y$ & mole fraction in the vapor phase \\
\hline Z & compressibility factor \\
\hline \multicolumn{2}{|c|}{ Greck letters } \\
\hline$\alpha$ & temperature-dependent function \\
\hline$\rho$ & density \\
\hline$\phi$ & fugacity coefficient for a pure tluid \\
\hline$\phi_{1}$ & fugacity coefficient for component $i$ in a mixture \\
\hline$\omega$ & acentric factor \\
\hline \multicolumn{2}{|l|}{ Subscripts } \\
\hline $\mathrm{c}$ & critical value \\
\hline i. $j$ & component $i$ or $j$ \\
\hline $\mathrm{m}$ & mixture property \\
\hline $\mathrm{R}$ & reduced property \\
\hline \multicolumn{2}{|l|}{ Superscripts } \\
\hline cal & calculated value \\
\hline $\exp$ & experimental value \\
\hline $\mathrm{L}$ & liquid phase \\
\hline V & vapor phase \\
\hline
\end{tabular}

\section{Acknowledgements}

The authors are grateful to the National Science Council, Republic of China for supporting this research. 


\section{References}

[1] R. Stryjek, J.H. Vera, PRSV: an improved Peng-Robinson equation of state for pure compounds and mixtures, Can. J. Chem. Eng. 64 (1986) 323-333.

[2] R. Stryjek, J.H. Vera, PRSV2: a cubic equation of state for accurate vapor-liquid equilibria calculations, Can. J. Chem. Eng. 64 (1986) 820-826.

[3] C.H. Twu, J.E. Coon, J.R. Cunningham, A new generalized alpha function for a cubic equation of state: Part 2. Redlich-Kwong equation, Fluid Phase Equilib. 105 (1995) 61-69.

[4] J.J. Martin, Cubic equations of state-which?, Ind. Eng. Chem. Fundam. 18 (1979) 81-97.

[5] A. Peneloux, E. Rauzy, R. Freze, A consistent correction for Redlich-Kwong-Soave volumes, Fluid Phase Equilib. 8 (1982) 7-23.

[6] G. Soave, Equilibrium constants from a modified Redlich-Kwong equation of state, Chem. Eng. Sci. 27 (1972) $1197-1203$.

[7] J.-M. Yu, B.C.-Y. Lu, A three-parameter cubic equation of state for asymmetric mixture density calculations, Fluid Phase Equilib. 34 (1987) 1-19.

[8] B. Carrier, M. Rogalski, A. Peneloux, Correlation and prediction of physical properties of hydrocarbons with the modified Peng-Robinson equation of state: 1. Low and medium vapor pressure, Ind. Eng. Chem. Res. 27 (1988) $1714-1721$

[9] M. Rogalski, B. Carrier, R. Solimando, A. Peneloux, Correlation and prediction of physical properties of hydrocarbons with the modified Peng-Robinson equation of state: 2. Representation of the vapor pressure and of the molar volume, Ind. Eng. Chem. Res. 29 (1990) 659-666.

[10] P. Watson, M. Cascella, D. May, S. Salerno, D. Tassios, Prediction of vapor pressures and saturated volumes with a simple cubic equation of state: Part II. The van der Waals-711 EOS, Fluid Phase Equilib. 27 (1986) 35-52.

[11] K. Magoulas, D. Tassios, Thermophysical properties of $n$-alkanes from $C_{1}$ to $C_{20}$ and their prediction for higher ones, Fluid Phase Equilib. 56 (1990) 119-140.

[12] M.A. Satyro, M.A. Trebble, On the applicability of the Sandler-Wong mixing rules for the calculation of thermodynamic excess properties $-V^{\mathrm{E}}, H^{\mathrm{E}}, S^{\mathrm{E}}, C_{p}^{\mathrm{E}}$, Fluid Phase Equilib. 115 (1996) 135-164.

[13] M.C. Kenney, V.S. Dodd, K.A. Smith, H.J. Herzog, J.W. Tester, A hard sphere volume translated van der Waals equation of state for supercritical process modeling, Fluid Phase Equilib. 128 (1997) 149-171.

[14] N.B. Vargaftik, Tables on the Thermophysical Properties of Liquids and Gases, Hemisphere, Washington, DC, 1975.

[15] D.P.M. Nunes, L.A.K. Staveley, The equation of state and thermodynamic properties of liquid hydrogen chloride, J. Chem. Thermodyn. 13 (1981) 179-186.

[16] B.D. Smith, R. Srivastava, Thermodynamic Data for Pure Compounds, Elsevier, Amsterdam, 1986.

[17] D.L. Morgan, R. Kobayashi, Direct vapor pressure measurements of ten $n$-alkanes in the $\mathrm{C}_{10}-\mathrm{C}_{28}$ range, Fluid Phase Equilib. 97 (1994) 211-242.

[18] C.F. Spencer, S. Alder, A critical review of equations for predicting saturated liquid density, J. Chem. Eng. Data 23 (1978) 82-89.

[19] R.D. Chirico, A. Nguyen, W.V. Steele, M.M. Strube, Vapor pressure of $n$-alkanes revisited. New high-precision vapor pressure data on $n$-decane, $n$-eicosane, and $n$-octacosane, J. Chem. Eng. Data 34 (1989) 149-156.

[20] F.D. Rossini, K.S. Pitzer, R.L. Arnett, R.M. Braun, G.C. Pimental, Selected values of properties of hydrocarbons and related compounds, API Project 44, Carnegie, Pittsburgh, 1952.

[21] H.S. Elbro, A. Fredenslund, P. Rasmussen, Group contribution method for the prediction of liquid densities as a function of temperature for solvents, oligomers, and polymers, Ind. Eng. Chem. Res. 30 (1991) 2576-2582.

[22] D. Ambrose, C.H.S. Sprake, R. Townsend, Thermodynamic properties of organic oxygen compounds: XXIX. The vapor pressure of diethyl ether, J. Chem. Thermodyn. 4 (1972) $247-254$.

[23] J.L. Hales, H.A. Gundry, J.H. Ellender, Liquid densities from 288 to $490 \mathrm{~K}$ of four organic oxygen compounds, J. Chem. Thermodyn. 15 (1983) 211-215.

[24] D. Ambrose, J.H. Ellender, C.H.S. Sprake, R. Townsend, Thermodynamic properties of organic oxygen compounds: XLII. Vapor pressures of some ethers, J. Chem. Thermodyn. 8 (1976) 165-178.

[25] TRC Thermodynamics Tables, Non-Hydrocarbons, Thermodynamics Research Center, The Texas A\&M University, College Station, TX, 1993. 
[26] D. Ambrose, J.H. Ellender, H.A. Gundry, D.A. Lee, R. Townsend, Thermodynamic properties of organic oxygen compounds: LI. The vapour pressures of some ethers and fatty acids, J. Chem. Thermodyn. 13 (1981) 795-802.

[27] E.F. Meyer, M.J. Awe, Cohesive energies in polar organic liquids: 4. n-alkvl acetates, J. Chem. Eng. Data 25 (1980) $371-374$.

[28] T. Boublik, V. Fried. E. Hala, The Vapor Pressures of Pure Substances, Elsevier, Amsterdam, 1973.

[29] J.A. Sandarusi, A.J. Kidnay, V.F. Yesavage, Compilation of parameters for a polar fluid Soave-Redlich-Kwong equation of state, Ind. Eng. Chem. Process Des. Dev. 25 (1986) 957-963.

[30] N.C. Patel, A.S. Teja, A new cubic equation of state for fluids and fluids mixtures, Chem. Eng. Sci. 37 (1982) $463-473$.

[3I] T.M. Guo, H. Kim, H.M. Lin, K.C. Chao, Cubic chain-of-rotators equation of state: 2. Polar substances, Ind. Eng. Chem. Process Des. Dev. 24 (1985) 764-767.

[32] T.M. Guo, H.M. Lin, K.C. Chao, Cubic chain-of-rotators equation of state: 3, Mixtures of polar substances, Ind. Eng. Chem. Process Des. Dev. 24 (1985) 768-773.

[33] H.T. Wang, J.C. Tsai, Y.P. Chen, A cubic equation of state for vapor-liquid equilibrium calculations of nonpolar and polar fluids, Fluid Phase Equilib. (1997) in press.

[34] R.C. Reid, J.M. Prausnitz, B.E. Polling, The Properties of Gases and Liquids, 4th edn., McGraw-Hill, New York, 1987.

[35] J. Gmehling, U. Onken, W. Arlt, P. Grenzheuser, U. Weidlich, B. Kolbe, J. Rarey-Nies, Vapor-Liquid Equilibrium Data Collection, DECHEMA, Frankfurt, Germany, 1978-date.

[36] J.R. Khurma, O. Muthu, S. Munjal, B.D. Smith, Total-pressure vapor-liquid equilibrium data for binary systems of dichloromethane with pentane, acetone, ethyl acetate, methanol and acetonitrile, J. Chem. Eng. Data 28 (1983) $412-419$.

[37] B.L. Larsen. P. Rasmussen, Aa. Fredenslund, A modified UNIFAC group-contribution model for prediction of phase equilibria and heats of mixing, Ind. Eng. Chem. Res. 26 (1987) 2274-2286.

[38] M.L. Michelsen, A method for incorporating excess Gibbs energy models in equation of state. Fluid Phase Equilib. 60 (1990) $47-58$.

[39] M.L. Michelsen, A modified Huron-Vidal mixing rule for cubic equations of state, Fluid Phase Equilib. 60 (1990) $213-219$.

[40] C. Boukouvalas, N. Spiliotis, Ph. Coutsikos, N. Tzouvaras, D.P. Tassios, Prediction of vapor-liquid equilibrium with the LCVM model: a linear combination of the Vidal and Michelsen mixing rules coupled with the original UNIFAC and the t-mPR equation of state, Fluid Phase Equilib. 92 (1994) 75-106.

[41] D.S.H. Wong, S.I. Sandler, A theoretically correct new mixing rule for cubic equation of state for both highly and slightly nonideal mixtures, AIChE J. 38 (5) (1992) 671-680.

[42] R. Srivastava, B.D. Smith, Total pressure vapor-liquid equilibrium data for binary systems of diethylamine with acetone, acetonitrile and methanol, J. Chem. Eng. Data 30 (1985) 308-313.

[43] J.L.E. Chevalier, P.J. Petrino, Y.H. Gaston-Bonhomme, Viscosity and density of some aliphatic, cyclic, and aromatic hydrocarbons binary liquid mixtures, J. Chem. Eng. Data 35 (1990) 206-212.

[44] A.A. Asfour, M.H. Siddique, Density-composition data for eight binary system containing toluene or ethylbenzene and C8-C16 n-alkanes at 293.15 K, 298.15 K,308.15 K, and 313.15 K, J. Chem. Eng. Data 35 (1990) 192-198.

[45] C. Franjo. E. Jimenez. T.P. Iglesias, J.L. Legido, M.I.P. Andrade, Viscosities and densities of hexane + I-butanol, +1 -hexanol, and +1-octanol at $298.15 \mathrm{~K}, \mathrm{~J}$. Chem. Eng. Data 40 (1995) 68-70.

[46] C. Franjo, E. Jimenez. T.P. Iglesias, J.L. Legido, M.I.P. Andrade, Viscosities and densities of octane + I-butanol, + 1-hexanol, and + 1-octanol at 298.15 K, J. Chem. Eng. Data 40 (1995) 992-994.

[47] A. Arce. A.S. Blanco, I. Vidal, Densities, refractive indices, and excess molar volumes of the ternary system water + methanol +1 -octanol and water + ethanol +1 -octanol and their binary mixtures at $298.15 \mathrm{~K}$. J. Chem. Eng. Data 38 (1993) 336-340.

[48] M. Dizechi. E. Marschail. Viscosity of some binary and ternary liquid mixtures, J. Chem. Eng. Data 27 (1982) $358-363$

[49] N. Voros. S. Stamataki, D.T. Tassios, Effect of translation on the prediction of saturated densities of binary mixtures with a Peng-Robinson equation of state, Fluid Phase Equilib. 96 (1994) 51-63.

[50] V.G. Niesen, Vapor + liquid equilibria and coexisting densities of carbon dioxide $+n$-butane at 311 to $395 \mathrm{~K}$. J. Chem. Thermodyn. 21 (1989) 915-923. 